\title{
Global Pandemic and Rapid New Product Development of Medical Products
}

MOOTHEDATH CHANDRAN KUMARI and BIRADAR SAGAR, Indian Institute of Science, Bangalore

New product development is a strenuous and long process for companies. When the COVID-19 disease turned into a global pandemic, governments announced the need for various medical products that were scarce and at the same time imposed lockdowns and trade restrictions. During this period, rapid new product development of medical products was attempted by many companies worldwide using their existing supply chains. Data of 240 companies from non-medical product-segment background launching new medical products were collected and analyzed. Two case studies of Indian companies responding to shortages of ventilators and PPE are presented. A critical reflection of a new way of new product development and cooperation across firms is discussed.

CCS Concepts: • Social and professional topics $\rightarrow$ Computing / technology policy; Government technology policy; Governmental regulations;

Additional Key Words and Phrases: COVID -19, industrial resilience, innovation, new product development, medical product design, global governance

ACM Reference format:

Moothedath Chandran Kumari and Biradar Sagar. 2020. Global Pandemic and Rapid New Product Development of Medical Products. Digit. Gov.: Res. Pract. 2, 2, Article 17 (December 2020), 38 pages.

https://doi.org/10.1145/3428492

\section{COVID 19 AND GOVERNMENT RESPONSE}

Severe Acute Respiratory Syndrome-Coronavirus 2 (SARS-CoV2) or Coronavirus Disease (COVID-19), is an infectious disease outbreak first reported in November-December 2019 that later turned into a global pandemic in a few months, contracted by 17.3 million to date [1]. According to the World Health Organization (WHO), "COVID-19 spreads primarily through droplets generated when an infected person coughs, sneezes or speaks. One could also become infected by touching a contaminated surface and then touching one's eyes, nose or mouth before washing hands" [2]. Therefore, the preventive measures from contracting the virus as advised by WHO are - to keep at least 1-m distance from others, frequently disinfect contaminated surfaces, clean hands regularly, and avoid touching the nose and mouth [2]. To date, there is no commercially available vaccine for COVID -19 that has been found effective in human trials.

Authors' addresses: M. C. Kumari and B. Sagar, Design Innovation Centre, Centre for Product Design and Manufacturing, Indian Institute of Science, Bangalore 560012, India; emails: \{kumarimc, sagarbiradar\}@iisc.ac.in.

Permission to make digital or hard copies of all or part of this work for personal or classroom use is granted without fee provided that copies are not made or distributed for profit or commercial advantage and that copies bear this notice and the full citation on the first page. Copyrights for components of this work owned by others than ACM must be honored. Abstracting with credit is permitted. To copy otherwise, or republish, to post on servers or to redistribute to lists, requires prior specific permission and/or a fee. Request permissions from permissions@acm.org.

(c) 2020 Association for Computing Machinery.

2639-0175/2020/12-ART17 \$15.00

https://doi.org/10.1145/3428492

Digital Government: Research and Practice, Vol. 2, No. 2, Article 17. Publication date: December 2020. 
COVID-19 spread globally across many countries in the initial months through international travelers, which influenced almost all nations and governments to curb international passenger flights by March 2020 [3]. Governments also responded to the pandemic by imposing quarantine of air travelers, cancelling visas of non-citizens to enter countries, and by announcing nationwide lockdowns. Lockdowns imposed many restrictions, including sealing of borders, transport and domestic travel restrictions, and shutdowns of schools, universities, trade, commercial establishments, marketplaces, factories, and offices. Other than essential and emergency services like hospitals, pharmacies, and supplies of essential goods, people were restricted to indoors during the governmentimposed lockdowns.

Different governments imposed lockdowns at varying stringencies [4]. Geographic boundaries became significant with these restrictions and acted as barriers for the movement of people. Boundaries at different scalesfrom national to regional to district to streets to buildings to individual rooms-emerged as a crucial parameter for containing infected people to limit the spread of the disease. When borders became a delineating element for restrictions on movement of people and goods, supply chains were disrupted locally and globally. A supply chain disruption is defined as an unplanned and unanticipated event that disrupts the normal flow of goods and materials within a supply chain $[5,6]$. The global supply chain disruptions due to COVID-19-related lockdowns affected many businesses, especially the international import/export of medical products needed for COVID-19 care itself. These include COVID-19 diagnostic testing kits, medicines, personal protective equipment (PPE) like coveralls, N95 masks, and so on, which were highly critical and urgent needs of many nations.

Hence, as the COVID-19 infectivity rate increased, governments decided to retain the COVID medical supplies they internally produced. Around 80 countries (as of April 22, 2020) introduced export prohibitions and restrictions to medical products and essentials for COVID-19 care to tackle their internal shortage. Among these products, export of face and eye protection products was prohibited by 73 countries, followed by the export of protective garments (50 countries), gloves (47 countries), sanitizers and disinfectants (28 countries), pharmaceuticals (20 countries), medical devices including ventilators (10 countries), and COVID-19 test kits (6 countries) [7]. For example, the US, UK, and India imposed restrictions on the export of PPE, COVID-19 test kits, and medicines [7].

A few months prior to the onset of the COVID-19 pandemic, medical products accounted for approximately $5 \%$ of the total world trade, including both imports and exports in 2019 [8]. Among these medical products, "medicines" represented 56\% of imports, followed by "medical supplies" (17\%), "medical equipment" (14\%), and "personal protective equipment" (13\%). The US, Germany, and China were the top three importers of medical products accounting for a third of total imports during 2017-2019 [8]. For exports, 40\% of personal protective product exports were from three countries-China, Germany, and the US-with China being the top exporter of face masks (25\% share) [8]. More than half the world's respirators and ventilators were exported by four countries-Singapore, US, Netherlands, and China [8]. It is evident that merely three to four countries were global leading exporters of medical products prior to the COVID-19 pandemic. But after the onset of the pandemic, and the sudden global trade restrictions due to it, the import-dependent countries for medical products were at dire shortage of essential COVID care products to meet their surging internal demands.

Nations and companies with global supply chains often have strategies and redundancies to address common supply chain disruption scenarios like natural calamities. However, a global pandemic as a supply chain disruption scenario and pandemic preparedness was new to most countries. This article will discuss the business responses to the shortage of essential COVID-19 care medical products induced by trade restrictions by different governments and how new ways of industrial collaboration scenarios can be observed during new product development amidst lockdowns.

\section{RESPONSE TO PANDEMIC BY INDUSTRIES AND RAPID NEW PRODUCT DEVELOPMENT}

As the supply chain disruptions and government-imposed lockdowns due to the pandemic affected companies' businesses as usual, many firms ventured to develop the medical products that are in shortage for COVID-19

Digital Government: Research and Practice, Vol. 2, No. 2, Article 17. Publication date: December 2020. 
care. The reasons for this new product development (NPD) by firms are many. The shortage of the medical products motivated some firms to produce them as a corporate social responsibility. For example, Lóreal produced hand sanitizers to provide it free of cost to hospitals, pharmacies, and so on (Table A.1, row 4). Some firms entered medical products segments identifying market shortage as a business opportunity. Example, Steelbird, an Indian motorcycle helmet manufacturer, entered the medical product segment by producing face shields (Table A.1, row 231). Governments demanded that private firms and state-owned corporations produce medical products like ventilators and PPE coveralls. For example, General Motors (GM), an American automobile company, was ordered by the US government to make 30,000 ventilators (Table A.1, row 98). Some firms started producing medical products to procure the government offered benefit to open their factories during the lockdown. For example, Nomasei, a luxury shoe brand, produced masks, as doing so allowed their workers to remain employed (Table A.1. row 15). Some firms produced medical products to keep their business afloat by monetizing existing production facilities. For example, Chinese automotive company BYD Co altered their production lines to produce surgical face masks while car sales dropped (Table A.1, row 232).

Therefore, many firms from non-medical product-segment backgrounds in different countries entered into medical product design and development. Figure 1 illustrates the relationship behind the events leading to firms from diverse product segments entering NPD of medical products.

A product development process in general involves six stages-planning, concept development, system level design, detail design, testing and refinement, and production ramp-up [9]. Product development times of different products vary from 1-4.5 years depending on complexity. Very few products can be developed in less than 1 year. For example, the development time of a simple two-component product, Stanley Tools Jobmaster Screwdriver, was 1 year, whereas that of a new printer (HP Desk-Jet Printer) was 1.5 years [9]. A new product opportunity also varies in value, and the risks increase as organizations navigate from existing to new solutions, markets, and product categories [8]. The COVID-19 pandemic necessitated rapid new product development with short product development time spans of 2-3 months, and many firms from different product-segment backgrounds entered a new product category of medical product segment.

To demonstrate the diversity of the original product-segment background of the firms who newly launched medical products for COVID-19, an indicative survey of new product launches of medical products over the period from March to July 2020 was carried out. Data of 240 non-medical product firms across 26 countries were collected from digital media press articles, social media posts, promotions, company websites, and Google search. The keywords used for Google search include, "list of companies manufacturing covid face shields and ventilators," "new covid products by automotive companies," "new covid products by retail company," and so on. After obtaining the company names, data were collected from each company's official websites. COVID-19 product launches by some of the top companies in the automotive and retail sectors were searched individually in Google search. For example, the keywords were "new covid products by Mercedes Benz," "new covid products by Nike," and so on. The data were collected over a period of 2 months, from May to July 2020, whilst the demand of medical products were surging and COVID-19 product launches were still ongoing. Hence, this is an indicative survey illustrating the trend of product launches taking place during this period.

The data of 240 companies showed they were from 21 different product-segment backgrounds, viz. aerospace, automotive, biotechnology, brewing, business and technology solutions, the chemical industry, conglomerate, consumer appliances, consumer goods, defense research, design studios, engineering and robotics, food processing, fuel-cell manufacturers, garment manufacturers, apparel retail, home automation, industrial safety equipment, medical equipment, software firms, and sporting goods. Of these, 114 companies were from India, and 127 from 25 countries. Fifty-nine percent of companies were more than 25 years old, and 13\% were start-ups of less than 5 years old.

There are seven main COVID-19 medical product categories where the most product launches have occurred: masks, ventilators, hand sanitizers, PPE coveralls, face shields, COVID diagnostic test kits, and UV-based disinfection products.

Digital Government: Research and Practice, Vol. 2, No. 2, Article 17. Publication date: December 2020. 


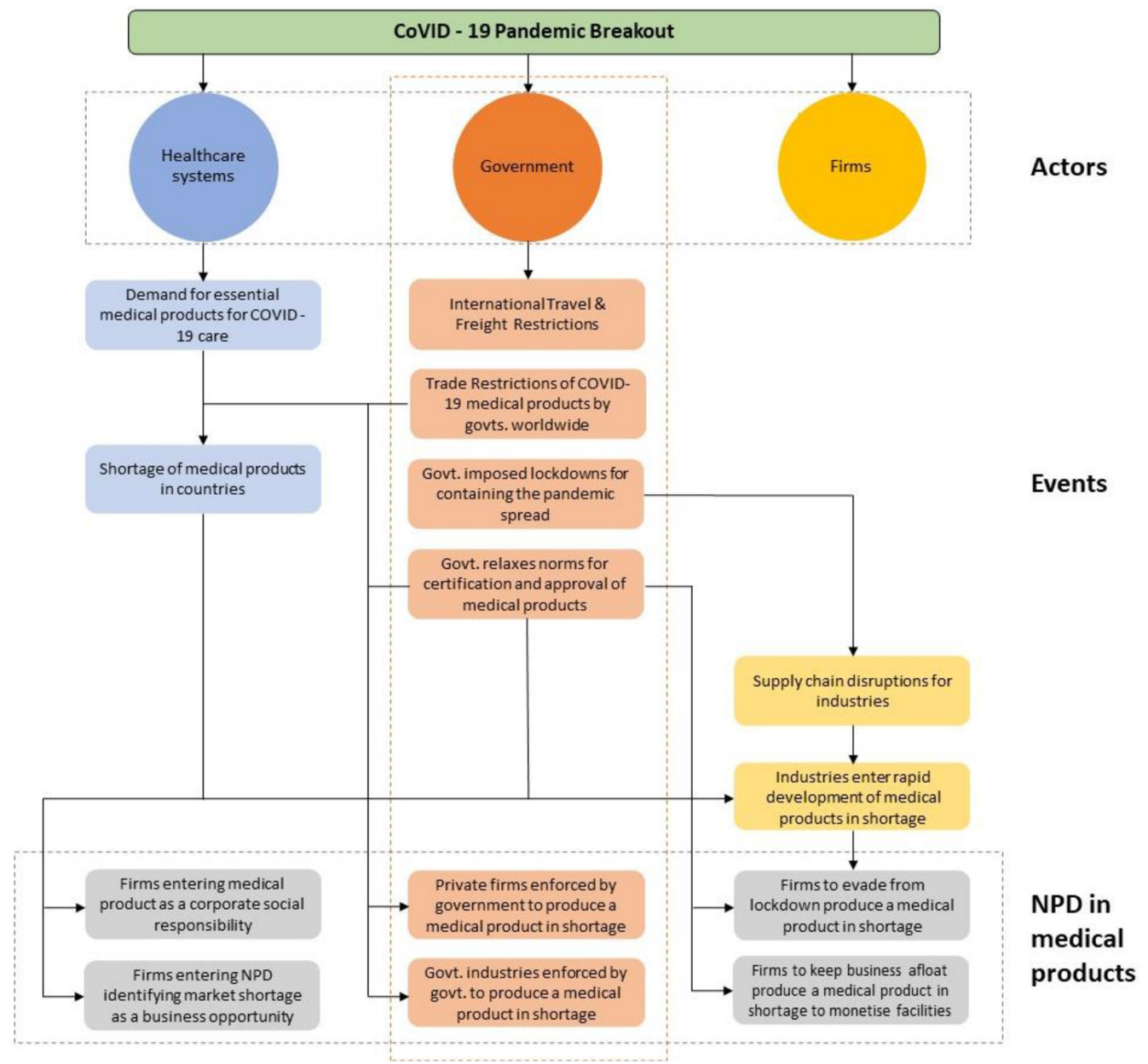

Fig. 1. Relationship between events leading to NPD in medical products by firms.

Figures 2 and 3 show the diversity of the original product-segment background of the firms that launched ventilator and PPE new products, respectively. Thirty-seven firms from 11 different product segments produced ventilators, and many of them are automotive and aerospace firms. As a ventilator is a product with mostly mechanical and electronic components, the automotive and aerospace companies would have had better access to raw material and component supply chains and manufacturing facilities for producing and assembling these components. New ventilator products were also launched by medical equipment firms, consumer appliances, consumer goods, and engineering and research organizations. Thirty-six firms from nine different product-segment backgrounds launched PPE products. While most of these firms are garment manufacturers and apparel retailers, automotive and consumer appliance firms also launched PPE products. This indicates that these firms adapted their current production systems to produce PPEs. The dataset is provided in Appendix A.1, and Appendix A.2 has bar charts for other COVID-19 medical products. We could see that the NPD of medical products were not limited to closely related product-segment firms, as many other firms from diverse product-segment backgrounds also launched COVID-19 medical products. Figure 4 illustrates this diversity of the original product-segment background of the firms versus the seven main COVID-19 medical products (masks, ventilators, hand sanitizers, 


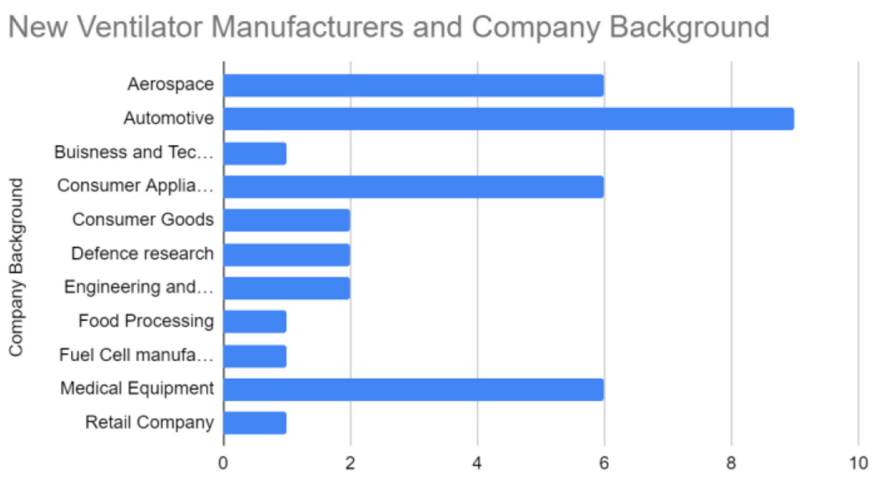

Fig. 2. Company backgrounds of 37 new ventilator producers.

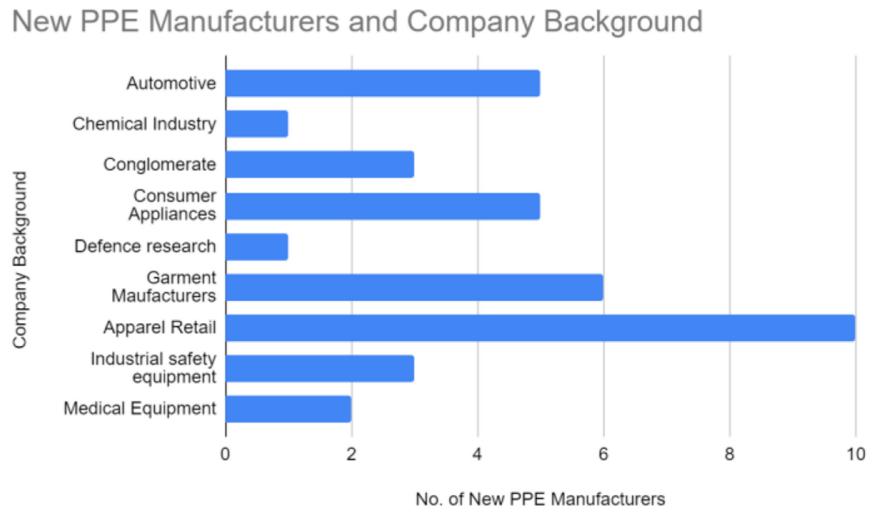

Fig. 3. Company backgrounds of 36 new PPE producers.

PPE coveralls, face shields, COVID diagnostic test kits, and UV-based disinfection products) for which NPD was carried out by firms.

\section{CASE STUDY OF RAPID NPD BY INDIAN INDUSTRIES AND THE ROLE OF GOVERNMENT OF INDIA}

When the Government of India (GoI) imposed a nationwide lockdown for 2 months from March 23, 2020 [10], India was facing shortage of PPEs, N95 masks, ventilators, and COVID diagnostic kits. On March 29, 2020, GoI appointed an Empowered Group (EG), who were entrusted with the responsibility of ensuring the availability of essential medical products necessary in India for the COVID-19 outbreak [11]. The EG was responsible for procurement, import, production, and distribution of PPEs, masks, gloves, and ventilators [11]. The EG took measures by reaching out to firms to produce these medical products in shortage within India and permitted firms producing medical products to open facilities during lockdowns with COVID prevention protocols. The GoI also waived a regulatory law that required a license for firms producing essential medical equipment if new manufacturers partnered with a licensed firm [12]. The Indian industries from different product-segment backgrounds responded to the government by entering NPD of essential medical products such as COVID diagnostic kits, PPEs, ventilators, masks, and face shields for rapid launch amidst lockdown. NPD amidst lockdown required government support for the necessary supply chains and logistics-interstate travel permits, local commute permits for employees, raw material shipping and sourcing assistance, and so on. The next section will discuss how 


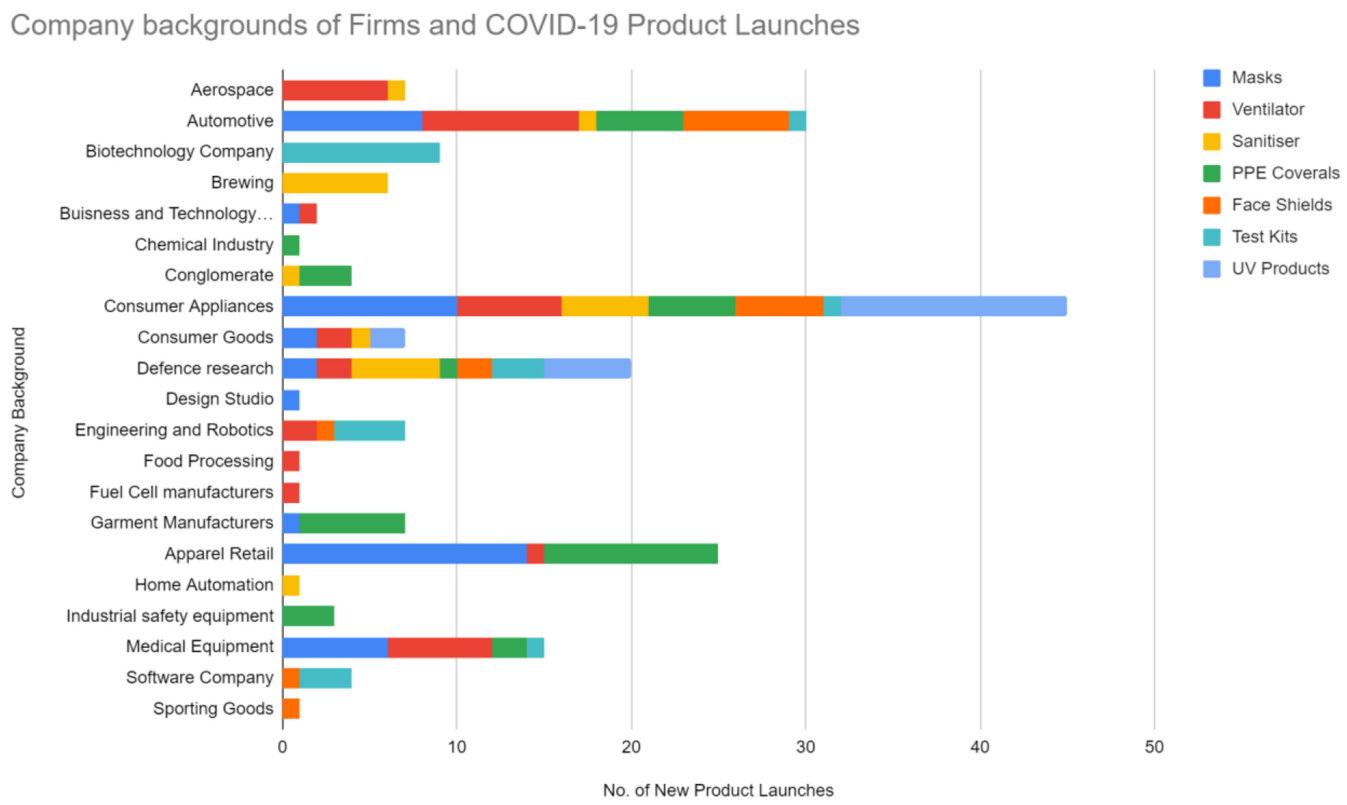

Fig. 4. Company backgrounds of 240 firms and new product categories.

companies collaborated with other firms or government organizations to execute an NPD process during supply chain disruptions. Two case studies of rapid NPD through collaboration by Indian firms responding to the shortage of medical products are presented below.

\subsection{Case Study 1: Company-driven Collaboration-Skanray Technologies + Mahindra \& Mahindra for Ventilator Design [12]}

Ventilators are necessary to treat critically ill COVID-19 patients facing breathing difficulties. India's domestic production capacity of ventilators was 2,500 units per month by seven companies in February 2020 until a month prior to the nationwide lockdown in March 2020. The Indian healthcare system was mainly relying on imported ventilators from Europe and China to meet the internal demands. In March 2020, the GoI projected an internal demand for 75,000 ventilators by June 2020 and imposed an export ban of ventilators. The EG placed orders for 60,884 ventilators to HLL Lifecare Limited (HLL), a GoI-owned corporation acting as a central procurement agency during the outbreak. HLL issued 59,884 orders to domestic manufacturers and 1,000 were to be imported.

Mahindra \& Mahindra (M\&M), an Indian automobile company, collaborated with Skanray Technologies (Skanray), an Indian medical equipment retail and original equipment manufacturer (OEM) company, to design and manufacture a new simplified ventilator design using easily available components and easy-to-use controls for its operation. Skanray shared the working knowledge of ventilators, and a new product design was developed that could be produced in M\&M's factories using existing facilities and shared components from its other product lines. The new product development stages are as follows:

- Concept Design: Skanray shared the technology and specifications of existing ventilator designs, and Mahindra identified components from its product platforms, and a new ventilator design concept was developed.

- Detailed design: M\&M’s mechanical and electronics engineers developed detailed design; software engineers wrote codes; and program managers and sourcing engineers prepared Bill of Materials and identified vendors.

Digital Government: Research and Practice, Vol. 2, No. 2, Article 17. Publication date: December 2020. 
- Procurement and Manufacturing: Mahindra was responsible for the procurement of components from its factories.

- Borrowed components for the new ventilator design from Mahindra's existing stock and facilities are as follows:

- Central Control - Borrowed from Mahindra Marazzo’s (MUV) compact multi-purpose vehicle platform.

- Control knobs- Borrowed from Mahindra Marazzo's Centre console switch bank and AC switch bank

- Displays for volume of gas, concentration of oxygen, error message - Borrowed from Mahindra Jawa's (motorcycle) round instrument cluster.

- Power back up - Borrowed from Mahindra Treo (electric powered three-wheeler rickshaw)

- Flow control valves - Skanray sourced from three suppliers (IMI Norgen, Parker, and Emerson) for redundancy to avoid supply delays during lockdown.

- Other parts - These were procured from more than 20 other suppliers who were helped by M\&M's taskforce to procure permission to open their facilities and to allow the necessary workforce to produce ventilator parts.

- Transportation and Logistics - Mahindra Logistics Ltd., the transport and supply chain logistics subsidiary of M\&M, brought all the parts from different facilities to Mahindra Research Valley, Chennai.

- Assembly - The ventilator was assembled at Mahindra Research Valley, Chennai.

- Prototype Testing - Pre-certification tests were carried out with guidance from Skanray.

The new ventilator was priced at 7500 INR, $0.75 \%$ is correct $(99.25 \%$ cheaper than the cost of an imported ventilator) [13].

\subsection{Case Study 2: Government-mediated Collaboration-Gol + Garment Manufacturers for PPE Design [14]}

India was import dependent for PPE kits (coveralls, N95 masks, gloves, and face and eye protection shields). In January 2020, India had only 0.275 million PPE kits in reserve, and 20 million was the projected demand in July 2020. Two of Gol's ministries, the Ministry of Textiles (MoT) and the Ministry of Health \& Family Welfare (MoHFW), collaborated and invited fabric and garment manufacturers to produce PPEs on a war-time basis. Companies who responded and offered their existing facilities to manufacture PPE were guided and assisted by MoT on PPE production and certifications. MoT tested fabric samples produced by textile manufacturers in India at a government-owned testing agency, South India Textile Research Association (SITRA), in Coimbatore. The results were shared with MoHFW, and technical specifications were finalized based on WHO interim guidelines on PPEs. An Emergency Control Room of the MoT centrally monitored the supply chain-sourcing raw materials and machines, production, and supply of medical textiles coordinating with the demands from governments. A government agency was assigned as a certifying body to test and certify new PPEs made by new firms. GoI further relaxed the testing standards to meet for PPEs. The Ministry of Civil Aviation supported the transportation of samples for testing and certification through state-owned airlines (Air India) during lockdown and ban on flights.

Arvind Mills, a textile and apparel company, entered NPD of PPE coveralls along with the local government of Ranchi city in the Indian state of Jharkhand as a public-private partnership. The NPD stages of PPE production of Arvind Mills and the Ranchi City Administration are [14,15] as follows:

- Raw materials: MoT assists in sourcing SITRA-approved fabrics through the Emergency Control Room.

- Procurement: Purchased by the District Administration, Ranchi, State of Jharkhand.

- PPE Coverall Design and technical guidance: Provided by MoT to Arvind Mills.

- Production: PPEs were produced at Arvind Mills Ranchi factory.

- Testing: SITRA, GoI-owned testing agency.

Digital Government: Research and Practice, Vol. 2, No. 2, Article 17. Publication date: December 2020. 
The PPEs were priced at 250 INR, at subsidized rates through the firm's CSR funds [15]. Arvind Mills now has a daily capacity of producing 15,000 PPE kits per day [14].

\section{CRITICAL REFLECTIONS ON THE ROLE OF GOVERNMENTS IN RAPID NPD AND COLLABORATION SCENARIOS}

The data of 240 companies in Section 2 illustrated the diversity of the product-segment background of the firms entering NPD of medical products during the pandemic. The case studies in Section 3 showed how two Indian firms from non-medical product backgrounds carried out NPD through strategic collaborations while the motivations for these collaborations were different. In the first case study, M\&M collaborated with an Indian medical equipment retail and OEM firm (Skanray) without a mandated government order to create ventilators. In the second case study from India, Arvind Mills was invited by the GoI and collaborated with the local government and the state-owned firms for PPE development and created a production capability. All partners in this alliance were new to medical products, except for the testing agency. In the US example of ventilator development by General Motors, GM collaborated with Ventec Life Systems, an American medical device company, to produce Ventec's ventilator design in GM's factories and was later mandated by the US government to deliver 30,000 ventilators [16, Table A.1, row 100]. These collaboration scenarios show the different motivations for firms to do NPD among those outlined in Section 2 and Figure 1. This section will discuss how the collaboration scenarios used by companies from different product-segment backgrounds for rapid NPD are different from other types of firm collaboration and co-operation alliances and the roles played by government in each of these.

Product and manufacturing firms cooperate with other firms for competitive advantage through strategic alliances. A strategic alliance is defined as an "independently initiated inter-firm link that involves exchange, sharing or co-development" [17 as in 18] or "contractual arrangements between two or more independent companies that carry out a project or operate in a specific business area by coordinating skills and resources jointly rather than either operating on their own or merging their operations" [19]. The different types of alliances from literature are as follows:

1. Exchange alliance - Resources are exchanged and utilized independently by each partner [18, 20].

2. Integration alliance - United resources shared in an organization designed by the partners to perform prescribed functions and to serve a common purpose $[18,20]$.

3. Symmetric alliance - The same kinds of resources are exchanged $[18,19]$.

4. Asymmetric alliance - Different kinds of resources are exchanged [18,19].

5. Horizontal alliance - Partners belong to the same industry [19].

6. Vertical alliance - Partners are from different industries [19].

Firms cooperate through different types or combinations of these alliances, and the motivations are different for each cooperation scenarios. We can identify the COVID NPD alliances as a combination of integration, asymmetric, and vertical alliance types. Below we will discuss about the different cooperation scenarios in product and manufacturing firms.

\subsection{Different Cooperation Scenarios in Product and Manufacturing Firms}

Resource-sharing is a supply chain cooperative action concept. It is commonly carried out in the field of transportation logistics. For example, in maritime shipping, companies optimize the utilization of freight carriers by using shared loads, groupage systems in freights in air transportation, and so on [21]. Resource sharing as one form of cooperation is also a beneficial way to react to supply chain disruptions [21]. Cooperating companies share machines for manufacturing to meet demand fluctuations by accessing each other's machines without maintaining redundancies and expensive capacities [21]. The partnership comes into force by market-driven

Digital Government: Research and Practice, Vol. 2, No. 2, Article 17. Publication date: December 2020. 
and economy-driven reasons, and decisions are taken within company borders. The cooperating partners may not be competitors.

Another concept involving cooperation between firms is industrial symbiosis (IS), which engages "traditionally separate industries in a collective approach to competitive advantage involving physical exchange of materials, energy, water, and/or by-products" (Reference [22], p. 313, as in Reference [23]). In IS, waste streams and by-products of one firm are used as inputs for other firms to generate economic benefits for the cooperating firms $[23,24]$. For example, a sugarcane refinery uses heat from the hot water used in combined heat and power plants to heat a glasshouse that grows tomatoes [25], which is not a primary product of the firm. Geographic proximity is often required in sharing resources for IS. The United Kingdom reports the highest number of IS cases, as the UK government launched the National Industrial Symbiosis Programme [26, 27], a voluntary program to help companies find partners that use their waste as raw material. China has highest number of IS cases in Asia, as the Chinese government has constraints on carbon dioxide emissions and plans and policies to foster circular economy practices [26, 28-30]. In Japan, IS is driven by the Japanese Eco-Town Programme, which encourages the use of waste in industrial applications to boost the economy and reduce waste disposal [26,31, 32].

Co-opetition is another supply chain cooperation concept in which independent companies simultaneously both cooperate and compete as a business constellation. It offers the opportunity of creating additional value for the cooperating firms [21,33]. For example, PSA group, a French automobile manufacturer, and Toyota, a Japanese automobile manufacturer, shared components for the PSA Peugeot 107, the Toyota Aygo, and the PSA Citroën C1 [34]. The companies saved on the shared production costs but remained competitive in other areas [34].

Platform-sharing is a product development method where brands share various products with the same architecture, components, technologies, and service procedures [35]. Platform product is built around pre-existing technological sub-systems [9]. An example is engine designs or steering wheel designs used across different vehicles in an automobile company. The goals of platform sharing are to reduce development and manufacturing costs and to create diverse product families to extend their brands [35-38]. Platform sharing can also be across firms to create technically identical products using different brand names. For example, the Suzuki Baleno 2015 model and its rebranded model, the Toyota Glanza, are both sold simultaneously in Indian market by Maruti Suzuki and Toyota's Indian subsidiary, Toyota Kirloskar [22]. Here the complete product is shared by two firms, and its combined market share is higher than its individual sale by Suzuki [22].

\subsection{Difference of COVID-19 NPD Alliance Co-operation Scenario}

Though the above four supply chain cooperation concepts share commonalities, there are differences in its goals and outputs. In resource-sharing, the participating companies do not have a common product development goal. In IS networks, the focus is on waste streams, and there is no guaranteed production of the co-product (product made from raw materials from waste streams of other products) in required capacities. The development of a coproduct is not a common goal of cooperating firms, but reduction of waste and carbon emissions are the goals, where in some cases it is driven by government laws and policies. Co-opetition involves different companies focusing on their individual products with some collaboration. Firms do not jointly carry out a new product development. Platform-sharing scenarios share designs of product sub-systems from one product to another, or entire product design from one firm to another, sharing resources and supply chains. The outcome is multiple products or the same product sold under different brand names, and both products are usually of the same product-segment.

In contrast to the above four co-operation scenarios, the COVID-19 NPD alliances shared resources to make a new product of a different domain as a shared goal. In Case Study 1 Ventilator Design of M\&M and Skanray (see Figure 5), M\&M shared parts from its three existing vehicles from three different product-segments, i.e., a motorcycle, a three-wheeler electric-powered rickshaw, and an MUV (minivan), which are not sharing platform

Digital Government: Research and Practice, Vol. 2, No. 2, Article 17. Publication date: December 2020. 


\section{Ventilator : Mahindra \& Mahindra + Skanray Technologies}

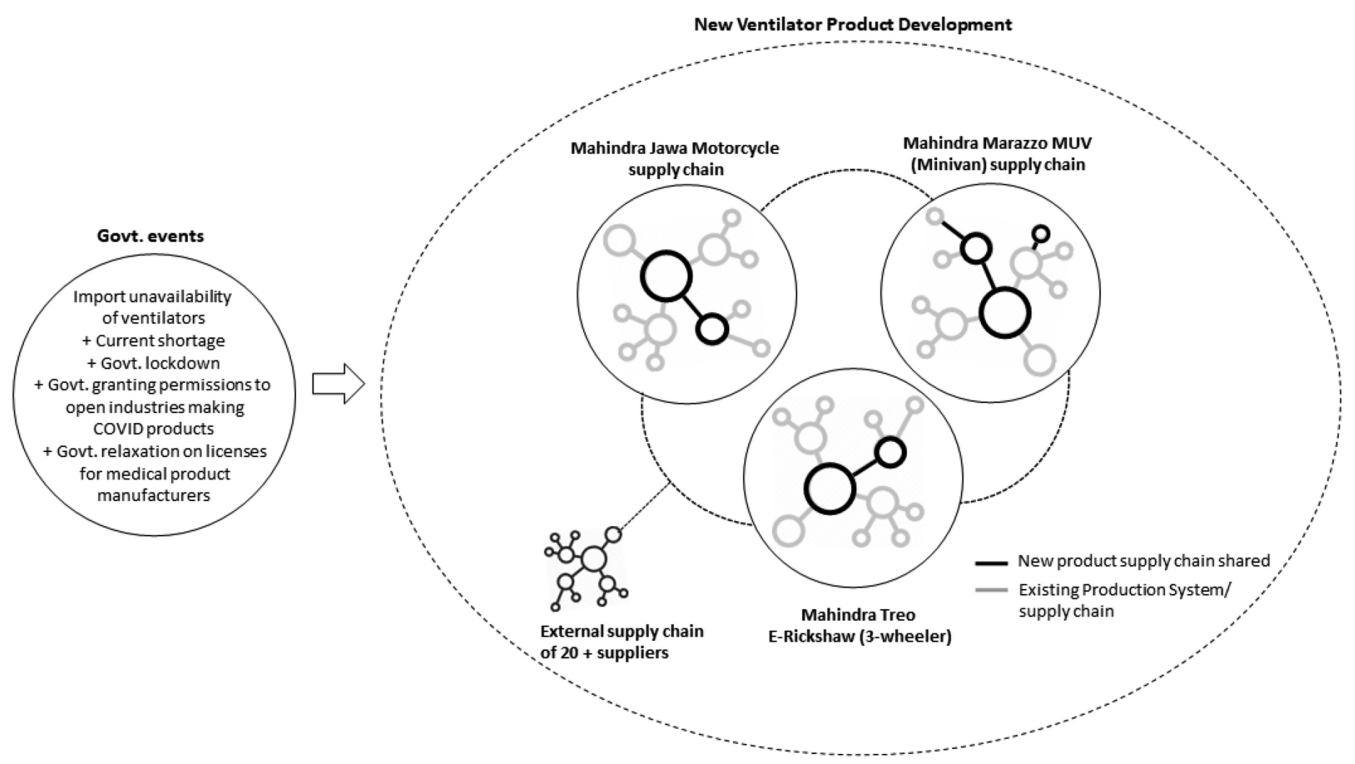

Fig. 5. New ventilator product supply chain from partly shared supply chains.

products among each other. Apart from these, a new supply chain was created with 20 other manufacturers. Together, a new product with a new supply chain was developed that shared three existing products' partial supply chain network. The two firms shared a single goal of one NPD that was neither an allied/substitute product of M\&M nor a by-product. Moreover, a large part of the workforce worked remotely, unlike other cooperation scenarios.

If we consider the area of supply chain resilience, according to Craighead et al. [6,40], then it is defined as the ability of a system to return to its original state or move to a new, more desirable state after being disturbed. The focus is on returning to the original state of equilibrium of the concerned supply chain. Resilience of industrial eco-systems concerns economic benefits as well as the reduction of material, energy, and environmental impacts. Though COVID-19 NPD had a common element of material and energy reduction by adapting existing facilities for new product development, reduction of environmental impact was not its main goal. It could be said that infectious disease is an environmental health hazard, but it is not a factor usually considered in resilience measures [40].

Table 1 summarizes the above co-operation concepts and situates the cooperation phenomenon observed in COVID-19 NPD alliances. From Table 1, we can see that the COVID-19 pandemic instigated NPD and manufacturing appears as a new firm supply chain cooperation scenario. A worldwide pandemic is also a new disaster scenario for supply chain disruption perhaps comparable to World War-II, which also instigated new technology developments in weaponry, vehicle designs, medicines, and packaged food [41]. Unlike other supply chain disruptive events during a natural calamity that are region specific, like typhoons, earthquakes, and floods, where firms respond by aid through CSR funds, for the COVID pandemic disaster scenario, firms entered NPD for the different reasons stated in Figure 1 and perhaps also from social responsibility, as 150 of 240 firms launched medical products amidst lockdown in their regions. The motivations of the 240 firms who launched medical products may differ from those of the case study examples, as the motivations per se are not captured in this study. The diversity of the companies entering medical product segment demonstrates the desire to act on a common goal of 
Table 1. Supply Chain Cooperation Scenarios for Multiple Products

\begin{tabular}{|c|c|c|c|c|c|}
\hline $\begin{array}{l}\text { Cooperation } \\
\text { scenarios }\end{array}$ & Description & Shared resource & $\begin{array}{l}\text { New Product } \\
\text { Domain }\end{array}$ & Goal & Role of governments \\
\hline $\begin{array}{l}\text { Resource- } \\
\text { sharing }\end{array}$ & $\begin{array}{l}\text { Cooperating } \\
\text { companies allow each } \\
\text { other to access its } \\
\text { resources }\end{array}$ & $\begin{array}{l}\text { Technical manpower, } \\
\text { manufacturing } \\
\text { facilities, supply } \\
\text { chains, } \\
\text { Intellectual property }\end{array}$ & $\begin{array}{l}\text { Same/ } \\
\text { different } \\
\text { domains }\end{array}$ & $\begin{array}{l}\text { To deal with supply } \\
\text { chain disruption or } \\
\text { other disturbing } \\
\text { events; Economic } \\
\text { benefits of sharing; } \\
\text { lean manufacturing }\end{array}$ & None \\
\hline $\begin{array}{l}\text { Industrial } \\
\text { Symbiosis } \\
\text { Network }\end{array}$ & $\begin{array}{l}\text { By-products from } \\
\text { waste streams are } \\
\text { shared as raw } \\
\text { materials to other } \\
\text { companies }\end{array}$ & Waste as raw material & $\begin{array}{l}\text { Same/ } \\
\text { different } \\
\text { domain }\end{array}$ & $\begin{array}{l}\text { Resource efficiency } \\
\text { and reducing wastage }\end{array}$ & $\begin{array}{l}\text { Benefits provided by } \\
\text { govt. as energy credits, } \\
\text { carbon credits }\end{array}$ \\
\hline Co-opetition & $\begin{array}{l}\text { Competing } \\
\text { companies making } \\
\text { similar products } \\
\text { collaborate and } \\
\text { compete at the same } \\
\text { time }\end{array}$ & $\begin{array}{l}\text { Product components, } \\
\text { supply chain }\end{array}$ & Same domain & $\begin{array}{l}\text { Combined Value } \\
\text { addition, lean }\end{array}$ & None \\
\hline $\begin{array}{l}\text { Platform } \\
\text { sharing }\end{array}$ & $\begin{array}{l}\text { Within or across } \\
\text { companies shared } \\
\text { resources for multiple } \\
\text { products }\end{array}$ & $\begin{array}{l}\text { Product component } \\
\text { designs, product } \\
\text { designs, intellectual } \\
\text { property }\end{array}$ & Same domain & $\begin{array}{l}\text { Economic and time to } \\
\text { market benefits; } \\
\text { resource efficiency; to } \\
\text { capture new markets }\end{array}$ & None \\
\hline $\begin{array}{l}\text { COVID-19 } \\
\text { NPD Alliance }\end{array}$ & $\begin{array}{l}\text { Multiple companies } \\
\text { shared different } \\
\text { resources and/or } \\
\text { build new supply } \\
\text { chains for making a } \\
\text { new product as a } \\
\text { common goal }\end{array}$ & $\begin{array}{l}\text { Product components, } \\
\text { machine, facility, } \\
\text { supply chain, } \\
\text { intellectual property }\end{array}$ & $\begin{array}{l}\text { Different } \\
\text { domain }\end{array}$ & $\begin{array}{l}\text { Socio-economic, } \\
\text { resource efficiency, } \\
\text { business, and } \\
\text { community survival } \\
\text { benefits. }\end{array}$ & $\begin{array}{l}\text { NPD mandated/promoted } \\
\text { by the govt.; NPD } \\
\text { instigated by supply } \\
\text { chain disruptions due to } \\
\text { govt. policies, trade } \\
\text { restrictions and } \\
\text { lock-down decisions; } \\
\text { Govt. relaxing licenses } \\
\text { and certifications for } \\
\text { medical product } \\
\text { manufacturing. }\end{array}$ \\
\hline
\end{tabular}

addressing a global pandemic. The case studies show that industrial or supply chain resilience cannot be looked at in isolation within company or alliance boundaries in the situation of a global pandemic. There is need for research on addressing supply chain disruptions due to a global pandemic and resource-constrained production where there is restriction on human proximity at workplaces.

\section{CONCLUSIONS}

A global pandemic of this scale of geographic spread at a time is the first of its kind. Human-made systems of supply chains, organizations, and work environments, including the way designers work, were affected as these structures changed. This paper discussed the rapid NPD of medical products by firms of different productsegment backgrounds and new firm cooperation scenarios that emerged due to constraints imposed by the pandemic as well as by the governmental actions of lockdowns and travel and export/import restrictions. The driving factor for firms to solve design problems and enter NPD of medical products for COVID-19 were multifacetedeconomic (demand for particular sets of medical products, business and supply chain disruptions), social (corporate responsibility to respond to larger needs of the society), and governmental (lockdowns, trade restrictions, mandated product development). For a globalized market to survive, a pandemic preparedness plan should not be a matter of governments alone-they need to be devised involving international trade bodies and organizations. 
Further research on the COVID-19 NPD alliances and cooperation scenarios would help in understanding these scenarios as a future way of collaboration for the NPD of critical medical products on demand.

\section{A APPENDICES}

\section{A.1 Dataset of 240 Companies That Launched COVID-19 Medical Products}

\begin{tabular}{|c|c|c|c|c|c|c|c|c|c|c|}
\hline No. & $\begin{array}{l}\text { Company } \\
\text { Name }\end{array}$ & Industry & $\begin{array}{l}\text { COVID-19 } \\
\text { product }\end{array}$ & Reference & $\begin{array}{c}\text { Date of } \\
\text { Reference }\end{array}$ & Country & $\begin{array}{l}\text { Release } \\
\text { Date }\end{array}$ & $\begin{array}{l}\text { Year of } \\
\text { Estb. }\end{array}$ & $\begin{array}{l}\text { Is Product } \\
\text { Released } \\
\text { During } \\
\text { Lockdown? } \\
\text { Yes/No/No } \\
\text { Data }\end{array}$ & URLs \\
\hline 1 & LVMH & Retail company & $\begin{array}{l}\text { Hand } \\
\text { Sanitizers }\end{array}$ & Link & $16-03-2020$ & France & $\begin{array}{l}\text { Not } \\
\text { mentioned }\end{array}$ & 1987 & No Data & $\begin{array}{l}\text { https://www.bbc.com/ } \\
\text { news/business-51868756 }\end{array}$ \\
\hline 2 & Foxconn & $\begin{array}{l}\text { Consumer } \\
\text { appliances }\end{array}$ & Face Masks & Link & $07-02-2020$ & Taiwan & $\begin{array}{c}\text { Not } \\
\text { mentioned }\end{array}$ & 1974 & No Data & $\begin{array}{l}\text { https://www.bbc.com/ } \\
\text { news/business- } 51410700\end{array}$ \\
\hline 3 & Welspun & Conglomerate & $\begin{array}{l}\text { Face Masks, } \\
\text { Disinfection } \\
\text { Wipes }\end{array}$ & Link & 07-04-2020 & India & $\begin{array}{c}\text { Not } \\
\text { mentioned }\end{array}$ & 1985 & No Data & $\begin{array}{l}\text { https://economictimes. } \\
\text { indiatimes.com/ } \\
\text { industry/cons-products/ } \\
\text { garments-/-textiles/ } \\
\text { welspun-to-start- } \\
\text { making-disinfectant- } \\
\text { wipes-masks/ } \\
\text { articleshow/75019409. } \\
\text { cms?from=mdr }\end{array}$ \\
\hline 4 & Loreal & Retail company & $\begin{array}{l}\text { Hand } \\
\text { Sanitizers }\end{array}$ & Link & $28-03-2020$ & France & $07-04-2020$ & 1909 & Yes & $\begin{array}{l}\text { https://economictimes. } \\
\text { indiatimes.com/ } \\
\text { magazines/panache/ } \\
\text { loreal-joins-fight- } \\
\text { against-coronavirus-to- } \\
\text { deliver-free-hand- } \\
\text { sanitisers-to-hospitals- } \\
\text { and-pharmacies/ } \\
\text { articleshow/74863953. } \\
\text { cms?from=mdr }\end{array}$ \\
\hline 5 & $\begin{array}{l}\text { La-Rochey- } \\
\text { Pose }\end{array}$ & Retail company & $\begin{array}{l}\text { Hydro- } \\
\text { alchoholic } \\
\text { gel }\end{array}$ & Link & $29-04-2020$ & France & $29-04-2020$ & 1976 & Yes & $\begin{array}{l}\text { https://fashionista.com/ } \\
\text { 2020/03/fashion-beauty- } \\
\text { companies-coronavirus- } \\
\text { covid19-manufacturing- } \\
\text { products-help }\end{array}$ \\
\hline 6 & $\begin{array}{l}\text { Americal } \\
\text { Apparels }\end{array}$ & Retail company & Face Masks & Link & $29-04-2020$ & USA & $29-04-2020$ & 1991 & Yes & $\begin{array}{l}\text { https://fashionista.com/ } \\
\text { 2020/03/fashion-beauty- } \\
\text { companies-coronavirus- } \\
\text { covid19-manufacturing- } \\
\text { products-help }\end{array}$ \\
\hline 7 & Zara & Retail company & $\begin{array}{l}\text { Hospital } \\
\text { Gowns, Face } \\
\text { Masks }\end{array}$ & Link & 29-04-2020 & Spain & $29-04-2020$ & 1974 & Yes & $\begin{array}{l}\text { https://fashionista.com/ } \\
\text { 2020/03/fashion-beauty- } \\
\text { companies-coronavirus- } \\
\text { covid19-manufacturing- } \\
\text { products-help }\end{array}$ \\
\hline 8 & Guerlain & Retail company & $\begin{array}{l}\text { Hand } \\
\text { Sanitizers }\end{array}$ & Link & 29-04-2020 & France & $29-04-2020$ & 1828 & Yes & $\begin{array}{l}\text { https://fashionista.com/ } \\
\text { 2020/03/fashion-beauty- } \\
\text { companies-coronavirus- } \\
\text { covid19-manufacturing- } \\
\text { products-help }\end{array}$ \\
\hline
\end{tabular}

Digital Government: Research and Practice, Vol. 2, No. 2, Article 17. Publication date: December 2020. 


\begin{tabular}{|c|c|c|c|c|c|c|c|c|c|c|}
\hline 9 & Big Bud Press & Retail company & Face Masks & Link & 29-04-2020 & USA & 29-04-2020 & 2015 & Yes & $\begin{array}{l}\text { https://fashionista.com/ } \\
\text { 2020/03/fashion-beauty- } \\
\text { companies-coronavirus- } \\
\text { covid19-manufacturing- } \\
\text { products-help }\end{array}$ \\
\hline 10 & $\begin{array}{l}\text { Christian } \\
\text { Siriano's } \\
\text { sewing team }\end{array}$ & Retail company & Face Masks & Link & 29-04-2020 & USA & 29-04-2020 & 2008 & Yes & $\begin{array}{l}\text { https://fashionista.com/ } \\
\text { 2020/03/fashion-beauty- } \\
\text { companies-coronavirus- } \\
\text { covid19-manufacturing- } \\
\text { products-help }\end{array}$ \\
\hline 11 & $\begin{array}{l}\text { Balenciaga and } \\
\text { Saint Laurent }\end{array}$ & Retail company & Face Masks & Link & 29-04-2020 & France & 29-04-2020 & 2001 & Yes & $\begin{array}{l}\text { https://fashionista.com/ } \\
\text { 2020/03/fashion-beauty- } \\
\text { companies-coronavirus- } \\
\text { covid19-manufacturing- } \\
\text { products-help }\end{array}$ \\
\hline 12 & Gucci & Retail company & $\begin{array}{l}\text { Surgical } \\
\text { Masks }\end{array}$ & Link & 29-04-2020 & Italy & 29-04-2020 & 1921 & Yes & $\begin{array}{l}\text { https://fashionista.com/ } \\
\text { 2020/03/fashion-beauty- } \\
\text { companies-coronavirus- } \\
\text { covid19-manufacturing- } \\
\text { products-help }\end{array}$ \\
\hline 13 & Prada & Retail company & $\begin{array}{l}\text { Face Masks, } \\
\text { PPE } \\
\text { Coveralls }\end{array}$ & Link & 29-04-2020 & Italy & 29-04-2020 & 1913 & Yes & $\begin{array}{l}\text { https://fashionista.com/ } \\
\text { 2020/03/fashion-beauty- } \\
\text { companies-coronavirus- } \\
\text { covid19-manufacturing- } \\
\text { products-help }\end{array}$ \\
\hline 14 & Nomasei & Retail company & Face Masks & Link & 29-04-2020 & Belgium & 29-04-2020 & 2016 & Yes & $\begin{array}{l}\text { https://fashionista.com/ } \\
\text { 2020/03/fashion-beauty- } \\
\text { companies-coronavirus- } \\
\text { covid19-manufacturing- } \\
\text { products-help }\end{array}$ \\
\hline 15 & $H \& M$ & Retail company & PPE Kit & Link & 22-03-2020 & Sweden & 29-04-2020 & 1947 & No Data & $\begin{array}{l}\text { https://hmgroup. } \\
\text { com/media/news/ } \\
\text { general-news-2020/h-m- } \\
\text { group-to-supply- } \\
\text { protective-equipment- } \\
\text { for-hospitals.html }\end{array}$ \\
\hline 16 & $\begin{array}{l}\text { Alabama } \\
\text { Chanin }\end{array}$ & Retail company & $\begin{array}{l}\text { Face Masks, } \\
\text { Hospital } \\
\text { gowns }\end{array}$ & Link & $29-04-2020$ & USA & 29-04-2020 & 2006 & Yes & $\begin{array}{l}\text { https://fashionista.com/ } \\
\text { 2020/03/fashion-beauty- } \\
\text { companies-coronavirus- } \\
\text { covid19-manufacturing- } \\
\text { products-help }\end{array}$ \\
\hline 17 & ForDays & Retail company & $\begin{array}{l}\text { Surgical } \\
\text { Masks } \\
\end{array}$ & Link & 06-04-2020 & Japan & 01-04-2020 & 2016 & No Data & $\begin{array}{l}\text { https://www.fordays.com/ } \\
\text { 5-smiley-mask-pack }\end{array}$ \\
\hline 18 & $\begin{array}{l}\text { Asimov } \\
\text { Robotics }\end{array}$ & $\begin{array}{l}\text { Engineering } \\
\text { and Robotics }\end{array}$ & $\begin{array}{l}\text { Disinfection } \\
\text { Robots }\end{array}$ & Link & $30-03-2020$ & India & $30-05-2020$ & 2012 & Yes & $\begin{array}{l}\text { https://economictimes. } \\
\text { indiatimes.com/news/ } \\
\text { science/robots-help- } \\
\text { combat-covid-19-in- } \\
\text { world-and-maybe-soon- } \\
\text { in-india-too/articleshow/ } \\
\text { 74893405.cms?from=mdr }\end{array}$ \\
\hline 19 & $\begin{array}{l}\text { Nocca } \\
\text { Robotics, IIT } \\
\text { Kanpur }\end{array}$ & $\begin{array}{l}\text { Engineering } \\
\text { and Robotics }\end{array}$ & Ventilators & Link & 08-05-2020 & India & $\begin{array}{c}\text { Not } \\
\text { mentioned }\end{array}$ & 2017 & No Data & $\begin{array}{l}\text { https://economictimes. } \\
\text { indiatimes.com/small-biz/ } \\
\text { startups/newsbuzz/ } \\
\text { indian-startup-nocca- } \\
\text { robotics-plans-to-ship- } \\
\text { covid-19-ventilator- } \\
\text { from-may-end/ } \\
\text { articleshow/75626184.cms }\end{array}$ \\
\hline 20 & \begin{tabular}{|l|} 
Aerobiosys \\
Innovations, \\
IIT Hyderabad
\end{tabular} & $\begin{array}{l}\text { Engineering } \\
\text { and Robotics }\end{array}$ & Ventilators & Link & 04-04-2020 & India & 02-04-2020 & 2018 & Yes & $\begin{array}{l}\text { https://iith.ac.in/assets/ } \\
\text { files/pdf/Social-and-R-D- } \\
\text { Initiatives-to-fight- } \\
\text { against-Covid-19.pdf }\end{array}$ \\
\hline
\end{tabular}

Digital Government: Research and Practice, Vol. 2, No. 2, Article 17. Publication date: December 2020. 


\begin{tabular}{|c|c|c|c|c|c|c|c|c|c|c|}
\hline 21 & $\begin{array}{l}\text { Agva } \\
\text { Healthcare }\end{array}$ & $\begin{array}{l}\text { Medical } \\
\text { equipment }\end{array}$ & Ventilators & Link & $30-06-2020$ & India & 02-04-2020 & 2017 & Yes & $\begin{array}{l}\text { https://www. } \\
\text { agvahealthcare.com/ }\end{array}$ \\
\hline 22 & $\begin{array}{l}\text { Indian Army's } \\
\text { Corps of } \\
\text { Electronics } \\
\text { and } \\
\text { Mechanical } \\
\text { Engineers } \\
\text { (EME) }\end{array}$ & $\begin{array}{l}\text { Defence } \\
\text { research }\end{array}$ & \begin{tabular}{|l} 
Remote \\
controlled \\
trolley; \\
Surgical \\
Masks, Hand \\
sanitizers, \\
Anti- \\
aerosolization \\
boxes, \\
Thermal \\
Scanners
\end{tabular} & Link & 03-05-2020 & India & $\begin{array}{c}\text { Not } \\
\text { mentioned }\end{array}$ & 1943 & No Data & $\begin{array}{l}\text { https://www. } \\
\text { indiatvnews.com/ } \\
\text { technology/news-tech- } \\
\text { innovations-by-indian- } \\
\text { army-drdo-to-fight- } \\
\text { coronavirus-pandemic- } \\
\text { covid-19-613697 }\end{array}$ \\
\hline 23 & $\begin{array}{l}\text { Centre for } \\
\text { Fire Explosive } \\
\text { \& } \\
\text { Environment } \\
\text { Safety } \\
\text { (CFEES), } \\
\text { Delhi }\end{array}$ & $\begin{array}{l}\text { Defence } \\
\text { research }\end{array}$ & $\begin{array}{l}\text { Sanitiser } \\
\text { Dispensing } \\
\text { Unit }\end{array}$ & Link & $03-05-2020$ & India & $\begin{array}{c}\text { Not } \\
\text { mentioned }\end{array}$ & 1992 & No Data & $\begin{array}{l}\text { https://www. } \\
\text { indiatvnews.com/ } \\
\text { technology/news-tech- } \\
\text { innovations-by-indian- } \\
\text { army-drdo-to-fight- } \\
\text { coronavirus-pandemic- } \\
\text { covid-19-613697 }\end{array}$ \\
\hline 24 & \begin{tabular}{l|} 
DIPAS, \\
INMAS, \\
DRDO \\
laboratories in \\
Delhi
\end{tabular} & $\begin{array}{l}\text { Defence } \\
\text { research }\end{array}$ & $\begin{array}{l}\text { UVC } \\
\text { Disinfection } \\
\text { Product }\end{array}$ & Link & 03-05-2020 & India & $\begin{array}{c}\text { Not } \\
\text { mentioned }\end{array}$ & 1961 & No Data & $\begin{array}{l}\text { https://www. } \\
\text { indiatvnews.com/ } \\
\text { technology/news-tech- } \\
\text { innovations-by-indian- } \\
\text { army-drdo-to-fight- } \\
\text { coronavirus-pandemic- } \\
\text { covid-19-613697 }\end{array}$ \\
\hline 25 & $\begin{array}{l}\text { Mylab } \\
\text { Discovery }\end{array}$ & $\begin{array}{l}\text { Biotechnology } \\
\text { company }\end{array}$ & Test Kits & Link & 04-06-2020 & India & 02-04-2020 & 2016 & Yes & $\begin{array}{l}\text { https://www. } \\
\text { industrialautomationindia. } \\
\text { in/startupsitm/9430/The- } \\
\text { Innovations-Indian- } \\
\text { Startups-are-Coming-up- } \\
\text { with-to-Combat-Covid- } \\
\text { 19/startups }\end{array}$ \\
\hline 26 & $\begin{array}{l}\text { A water } \\
\text { treatment } \\
\text { company }\end{array}$ & $\begin{array}{l}\text { Biotechnology } \\
\text { company }\end{array}$ & $\begin{array}{l}\text { Disinfection } \\
\text { Tunnel }\end{array}$ & Link & $04-06-2020$ & India & $02-04-2020$ & 2017 & Yes & $\begin{array}{l}\text { https://www. } \\
\text { industrialautomationindia. } \\
\text { in/startupsitm/9430/The- } \\
\text { Innovations-Indian- } \\
\text { Startups-are-Coming-up- } \\
\text { with-to-Combat-Covid- } \\
\text { 19/startups }\end{array}$ \\
\hline 27 & $\begin{array}{l}\text { Turtle Shell } \\
\text { Technologies }\end{array}$ & $\begin{array}{l}\text { Biotechnology } \\
\text { company }\end{array}$ & $\begin{array}{l}\text { Health } \\
\text { Monitoring } \\
\text { Device }\end{array}$ & Link & 05-03-2020 & India & 05-03-2020 & 2015 & Yes & $\begin{array}{l}\text { https://www. } \\
\text { bloombergquint.com/ } \\
\text { business/startup-street- } \\
\text { dozees-contactless- } \\
\text { monitor-is-limiting- } \\
\text { frontline-health-workers- } \\
\text { exposure-to-covid-19 }\end{array}$ \\
\hline 28 & Qure.ai & $\begin{array}{l}\text { Biotechnology } \\
\text { company }\end{array}$ & PPE Kit & Link & $02-04-2020$ & India & $02-04-2020$ & 2016 & Yes & $\begin{array}{l}\text { https://economictimes. } \\
\text { indiatimes.com/startups/ } \\
\text { healthcare-startups- } \\
\text { dozee-qure-ai-log-9- } \\
\text { mylab-speed-up-efforts- } \\
\text { to-fight-covid-19/ } \\
\text { articleshow/74939112.cms }\end{array}$ \\
\hline 29 & Healthians & $\begin{array}{l}\text { Biotechnology } \\
\text { company }\end{array}$ & Chatbot App & Link & $28-03-2020$ & India & $\begin{array}{c}\text { Not } \\
\text { mentioned }\end{array}$ & 2014 & No Data & $\begin{array}{l}\text { http://www. } \\
\text { businessworld.in/article/ } \\
\text { Indian-HealthTech- } \\
\text { Startups-leveraging- } \\
\text { Tech-to-tackle-COVID- } \\
\text { 19/28-03-2020-187513/ }\end{array}$ \\
\hline 30 & InAccel & $\begin{array}{l}\text { Medical } \\
\text { equipment }\end{array}$ & $\begin{array}{l}\text { Ventilator for } \\
\text { babies }\end{array}$ & Link & 08-04-2020 & India & $4 / 1 / 2020$ & 2012 & Yes & $\begin{array}{l}\text { https://inc42. } \\
\text { com/features/ } \\
\text { startupsvscovid19-indias- } \\
\text { medical-devices-startups- } \\
\text { step-up-to-the- } \\
\text { challenge/ }\end{array}$ \\
\hline
\end{tabular}

Digital Government: Research and Practice, Vol. 2, No. 2, Article 17. Publication date: December 2020. 


\begin{tabular}{|c|c|c|c|c|c|c|c|c|c|c|}
\hline 31 & InAccel & $\begin{array}{l}\text { Medical } \\
\text { equipment }\end{array}$ & $\begin{array}{l}\text { Non-Invasive } \\
\text { Ventilator }\end{array}$ & Link & 08-04-2020 & India & $7 / 21 / 2020$ & 2012 & No & $\begin{array}{l}\text { https://inc42.com/features/ } \\
\text { startupsvscovid19-indias- } \\
\text { medical-devices-startups- } \\
\text { step-up-to-the-challenge/ }\end{array}$ \\
\hline 32 & InAccel & $\begin{array}{l}\text { Medical } \\
\text { equipment }\end{array}$ & \begin{tabular}{|l} 
Automated \\
Oral \\
Secretion \\
Remover \\
\end{tabular} & Link & 08-04-2020 & India & $\begin{array}{l}\text { Not } \\
\text { mentioned }\end{array}$ & 2012 & No Data & $\begin{array}{l}\text { https://inc42.com/features/ } \\
\text { startupsvscovid19-indias- } \\
\text { medical-devices-startups- } \\
\text { step-up-to-the-challenge/ }\end{array}$ \\
\hline 33 & $\begin{array}{l}\text { Ashok } \\
\text { Leyland Ltd. }\end{array}$ & Automotive & $\begin{array}{l}\text { Face Masks, } \\
\text { Face Shield }\end{array}$ & Link & $31-03-2020$ & India & $05-05-2020$ & 1948 & Yes & $\begin{array}{l}\text { https://economictimes. } \\
\text { indiatimes.com/news/ } \\
\text { company/corporate-trends/ } \\
\text { tvs-motor-company- } \\
\text { ashok-leyland-nippon- } \\
\text { paints-among-others- } \\
\text { contribute-to-covid-19- } \\
\text { relief/articleshow/74912961. } \\
\text { cms?from=mdr }\end{array}$ \\
\hline 34 & $\begin{array}{l}\text { Ashok } \\
\text { Leyland Ltd. }\end{array}$ & Automotive & Ventilators & Link & 05-05-2020 & India & 05-05-2020 & 1948 & No Data & $\begin{array}{l}\text { https://www.thehindu.com// } \\
\text { business/ashok-leyland- } \\
\text { develops-low-cost- } \\
\text { ventilator/article31512380. } \\
\text { ece }\end{array}$ \\
\hline 35 & ORBIT Gear & $\begin{array}{l}\text { Engineering } \\
\text { and } \\
\text { Technology }\end{array}$ & Face Shield & Link & $10-05-2020$ & Indonesia & $10-05-2020$ & 2008 & No Data & $\begin{array}{l}\text { https://hypebeast.com/ } \\
\text { 2020/5/orbitgear-w210-a- } \\
\text { face-shield-release }\end{array}$ \\
\hline 36 & $\begin{array}{l}\text { Reliance } \\
\text { foundation }\end{array}$ & $\begin{array}{l}\text { Consumer } \\
\text { appliances }\end{array}$ & $\begin{array}{l}\text { Face Masks, } \\
\text { PPE Kits }\end{array}$ & Link & $23-03-2020$ & India & $23-03-2020$ & 2010 & No Data & $\begin{array}{l}\text { https://www.bignews } \\
\text { network.com/news/2643 } \\
98187 / \text { reliance-boosts- } \\
\text { production-of-face-masks- } \\
\text { to-one-lakh-per-day }\end{array}$ \\
\hline 37 & $\begin{array}{l}\text { Mahindra } \\
\text { and } \\
\text { Mahindra }\end{array}$ & Automotive & Face Shield & Link & $29-03-2020$ & India & $29-03-2020$ & 1945 & No Data & $\begin{array}{l}\text { https://auto.economictimes. } \\
\text { indiatimes.com/news/ } \\
\text { passenger-vehicle/cars/ } \\
\text { covid-19-fight-mahindra- } \\
\text { mahindra-to-make-face- } \\
\text { shield-for-health-workers- } \\
\text { pawan-goenka-reveals- } \\
\text { design/74869559 }\end{array}$ \\
\hline 38 & Xiaomi & $\begin{array}{l}\text { Consumer } \\
\text { appliances }\end{array}$ & N95 Masks & Link & $26-03-2020$ & China & $26-03-2020$ & 2010 & No Data & $\begin{array}{l}\text { https://indianexpress.com/ } \\
\text { article/technology/tech- } \\
\text { news-technology/vivo- } \\
\text { huami-and-xiaomi- } \\
\text { distributing-n95-masks- } \\
\text { medical-supplies-to- } \\
\text { indian-government- } \\
\text { authorities-6331155/ }\end{array}$ \\
\hline 39 & Diageo & Brewing & $\begin{array}{l}\text { Hand } \\
\text { Sanitizers }\end{array}$ & Link & $23-03-2020$ & England & $\begin{array}{l}\text { Not } \\
\text { mentioned }\end{array}$ & 1997 & No Data & $\begin{array}{l}\text { https://www.diageo.com/ } \\
\text { en/news-and-media/press- } \\
\text { releases/diageo-pledges- } \\
\text { more-than-eight-million- } \\
\text { bottles-of-sanitiser-for- } \\
\text { frontline-healthcare- } \\
\text { workers/ }\end{array}$ \\
\hline 40 & DRDO-VRDE & $\begin{array}{l}\text { Defence } \\
\text { research }\end{array}$ & \begin{tabular}{|l} 
Disinfection \\
Tunnel
\end{tabular} & Link & $04-05-2020$ & India & $14-04-2020$ & 1965 & Yes & $\begin{array}{l}\text { https://drdo.gov.in/counter- } \\
\text { covid-19-technologies }\end{array}$ \\
\hline 41 & DRDO-VRDE & $\begin{array}{l}\text { Defence } \\
\text { research }\end{array}$ & \begin{tabular}{|l} 
Personal \\
Sanitization \\
Unit \\
\end{tabular} & Link & $04-05-2020$ & India & $14-04-2020$ & 1965 & Yes & $\begin{array}{l}\text { https://drdo.gov.in/counter- } \\
\text { covid-19-technologies }\end{array}$ \\
\hline 42 & DRDO-DLJ & $\begin{array}{l}\text { Defence } \\
\text { research }\end{array}$ & \begin{tabular}{|l} 
Hand \\
Sanitizers
\end{tabular} & Link & 03-04-2020 & India & $14-04-2020$ & 1958 & Yes & $\begin{array}{l}\text { https://drdo.gov.in/counter- } \\
\text { covid-19-technologies }\end{array}$ \\
\hline 43 & DRDO-DLJ & $\begin{array}{l}\text { Defence } \\
\text { research }\end{array}$ & $\begin{array}{l}\text { Mobile Area } \\
\text { Sanitization } \\
\text { System }\end{array}$ & Link & 03-04-2020 & India & $14-04-2020$ & 1958 & Yes & $\begin{array}{l}\text { https://drdo.gov.in/counter- } \\
\text { covid-19-technologies }\end{array}$ \\
\hline
\end{tabular}

Digital Government: Research and Practice, Vol. 2, No. 2, Article 17. Publication date: December 2020. 


\begin{tabular}{|c|c|c|c|c|c|c|c|c|c|c|}
\hline 44 & $\begin{array}{l}\text { DRDO- } \\
\text { DEBEL }\end{array}$ & $\begin{array}{l}\text { Defence } \\
\text { research }\end{array}$ & $\begin{array}{l}\text { Single Outlet } \\
\text { Automatic } \\
\text { Resuscitator }\end{array}$ & Link & 09-06-2020 & India & $14-04-2020$ & 1958 & Yes & $\begin{array}{l}\text { https://drdo.gov. } \\
\text { in/counter-covid-19- } \\
\text { technologies }\end{array}$ \\
\hline 45 & $\begin{array}{l}\text { DRDO- } \\
\text { DEBEL }\end{array}$ & $\begin{array}{l}\text { Defence } \\
\text { research }\end{array}$ & $\begin{array}{l}\text { Medical- } \\
\text { Oxygen- } \\
\text { Plant, } \\
\text { Ventilatorz }\end{array}$ & Link & 09-06-2020 & India & $14-04-2020$ & 1958 & Yes & $\begin{array}{l}\text { https://drdo.gov. } \\
\text { in/counter-covid-19- } \\
\text { technologies }\end{array}$ \\
\hline 46 & $\begin{array}{l}\text { DRDO- } \\
\text { CFEES }\end{array}$ & $\begin{array}{l}\text { Defence } \\
\text { research }\end{array}$ & $\begin{array}{l}\text { Backpack } \\
\text { Santization } \\
\text { Equipment }\end{array}$ & Link & 03-04-2020 & India & $14-04-2020$ & 1992 & Yes & $\begin{array}{l}\text { https://drdo.gov. } \\
\text { in/counter-covid-19- } \\
\text { technologies }\end{array}$ \\
\hline 47 & $\begin{array}{l}\text { DRDO- } \\
\text { CFEES }\end{array}$ & $\begin{array}{l}\text { Defence } \\
\text { research }\end{array}$ & $\begin{array}{l}\text { Area } \\
\text { Sanitization } \\
\text { System }\end{array}$ & Link & 03-04-2020 & India & $14-04-2020$ & 1992 & Yes & $\begin{array}{l}\text { https://drdo.gov. } \\
\text { in/counter-covid-19- } \\
\text { technologies }\end{array}$ \\
\hline 48 & $\begin{array}{l}\text { DRDO- } \\
\text { CFEES }\end{array}$ & $\begin{array}{l}\text { Defence } \\
\text { research }\end{array}$ & $\begin{array}{l}\text { Sanitiser } \\
\text { Dispensing } \\
\text { Unit }\end{array}$ & Link & 03-04-2020 & India & $14-04-2020$ & 1992 & Yes & $\begin{array}{l}\text { https://drdo.gov. } \\
\text { in/counter-covid-19- } \\
\text { technologies }\end{array}$ \\
\hline 49 & $\begin{array}{l}\text { DRDO- } \\
\text { CFEES }\end{array}$ & $\begin{array}{l}\text { Defence } \\
\text { research }\end{array}$ & $\begin{array}{l}\text { Personal } \\
\text { Sanitization } \\
\text { Unit }\end{array}$ & Link & 03-04-2020 & India & $14-04-2020$ & 1992 & Yes & $\begin{array}{l}\text { https://drdo.gov. } \\
\text { in/counter-covid-19- } \\
\text { technologies }\end{array}$ \\
\hline 50 & DRDO & $\begin{array}{l}\text { Defence } \\
\text { research }\end{array}$ & \begin{tabular}{|l} 
UVC \\
Disinfection \\
Product
\end{tabular} & Link & 04-05-2020 & India & $14-04-2020$ & 1958 & Yes & $\begin{array}{l}\text { https://drdo.gov. } \\
\text { in/counter-covid-19- } \\
\text { technologies }\end{array}$ \\
\hline 51 & $\begin{array}{l}\text { INMAS, } \\
\text { Delhi }\end{array}$ & $\begin{array}{l}\text { Defence } \\
\text { research }\end{array}$ & $\begin{array}{l}\text { Disinfection } \\
\text { Tunnel }\end{array}$ & Link & $04-05-2020$ & India & $14-04-2020$ & 1961 & Yes & $\begin{array}{l}\text { https://drdo.gov. } \\
\text { in/counter-covid-19- } \\
\text { technologies }\end{array}$ \\
\hline 52 & $\begin{array}{l}\text { INMAS, } \\
\text { Delhi }\end{array}$ & $\begin{array}{l}\text { Defence } \\
\text { research }\end{array}$ & $\begin{array}{l}\text { Pen and } \\
\text { Pocket } \\
\text { Sanitizer }\end{array}$ & Link & 03-06-2020 & India & $14-04-2020$ & 1961 & Yes & $\begin{array}{l}\text { https://drdo.gov. } \\
\text { in/counter-covid-19- } \\
\text { technologies }\end{array}$ \\
\hline 53 & $\begin{array}{l}\text { INMAS, } \\
\text { Delhi }\end{array}$ & $\begin{array}{l}\text { Defence } \\
\text { research }\end{array}$ & $\begin{array}{l}\text { Sanitizing } \\
\text { Car Mat, } \\
\text { Sanitization } \\
\text { Shoe Mat }\end{array}$ & Link & $03-06-2020$ & India & $14-04-2020$ & 1961 & Yes & $\begin{array}{l}\text { https://drdo.gov. } \\
\text { in/counter-covid-19- } \\
\text { technologies }\end{array}$ \\
\hline 54 & $\begin{array}{l}\text { INMAS, } \\
\text { Delhi }\end{array}$ & $\begin{array}{l}\text { Defence } \\
\text { research }\end{array}$ & $\begin{array}{l}\text { UVC } \\
\text { Disinfection } \\
\text { Product }\end{array}$ & Link & $03-06-2020$ & India & $14-04-2020$ & 1961 & Yes & $\begin{array}{l}\text { https://drdo.gov. } \\
\text { in/counter-covid-19- } \\
\text { technologies }\end{array}$ \\
\hline 55 & $\begin{array}{l}\text { INMAS, } \\
\text { Delhi }\end{array}$ & $\begin{array}{l}\text { Defence } \\
\text { research }\end{array}$ & $\begin{array}{l}\text { PPE Testing } \\
\text { and } \\
\text { Certification }\end{array}$ & Link & 03-06-2020 & India & $14-04-2020$ & 1961 & Yes & $\begin{array}{l}\text { https://drdo.gov. } \\
\text { in/counter-covid-19- } \\
\text { technologies }\end{array}$ \\
\hline 56 & DRDO & $\begin{array}{l}\text { Defence } \\
\text { research }\end{array}$ & $\begin{array}{l}\text { Mobile } \\
\text { Diagnosis } \\
\text { Lab } \\
\end{array}$ & Link & $23-04-2020$ & India & $14-04-2020$ & 1958 & Yes & $\begin{array}{l}\text { https://drdo.gov. } \\
\text { in/counter-covid-19- } \\
\text { technologies }\end{array}$ \\
\hline 57 & CFEES & $\begin{array}{l}\text { Defence } \\
\text { research }\end{array}$ & \begin{tabular}{|l} 
Sanitiser \\
Dispensing \\
Unit \\
\end{tabular} & Link & $17-04-2020$ & India & $14-04-2020$ & 1992 & Yes & $\begin{array}{l}\text { https://drdo.gov. } \\
\text { in/counter-covid-19- } \\
\text { technologies }\end{array}$ \\
\hline 58 & DIPAS & $\begin{array}{l}\text { Defence } \\
\text { research }\end{array}$ & \begin{tabular}{|l} 
UVC \\
Disinfection \\
Product
\end{tabular} & Link & $17-04-2020$ & India & $14-04-2020$ & 1962 & Yes & $\begin{array}{l}\text { https://drdo.gov. } \\
\text { in/counter-covid-19- } \\
\text { technologies }\end{array}$ \\
\hline 59 & DRDO & $\begin{array}{l}\text { Defence } \\
\text { research }\end{array}$ & $\begin{array}{l}\text { Mobile } \\
\text { Diagnosis } \\
\text { Lab } \\
\end{array}$ & Link & $23-04-2020$ & India & $14-04-2020$ & 1958 & Yes & $\begin{array}{l}\text { https://drdo.gov. } \\
\text { in/counter-covid-19- } \\
\text { technologies }\end{array}$ \\
\hline 60 & $\begin{array}{l}\text { LASTEC, } \\
\text { Delhi }\end{array}$ & $\begin{array}{l}\text { Defence } \\
\text { research }\end{array}$ & $\begin{array}{l}\text { UVC } \\
\text { Disinfection } \\
\text { Product }\end{array}$ & Link & $04-05-2020$ & India & $14-04-2020$ & 1950 & Yes & $\begin{array}{l}\text { https://drdo.gov. } \\
\text { in/counter-covid-19- } \\
\text { technologies }\end{array}$ \\
\hline 61 & $\begin{array}{l}\text { CAIR, } \\
\text { Bengaluru }\end{array}$ & $\begin{array}{l}\text { Defence } \\
\text { research }\end{array}$ & $\begin{array}{l}\text { Robot for } \\
\text { Medical Use }\end{array}$ & Link & $28-05-2020$ & India & $14-04-2020$ & 1986 & Yes & $\begin{array}{l}\text { https://drdo.gov. } \\
\text { in/counter-covid-19- } \\
\text { technologies }\end{array}$ \\
\hline
\end{tabular}

Digital Government: Research and Practice, Vol. 2, No. 2, Article 17. Publication date: December 2020. 


\begin{tabular}{|c|c|c|c|c|c|c|c|c|c|c|}
\hline 62 & $\begin{array}{l}\text { TBRL- } \\
\text { Chandigarh }\end{array}$ & $\begin{array}{l}\text { Defence } \\
\text { research }\end{array}$ & $\begin{array}{l}\text { Hand } \\
\text { Sanitizer, } \\
\text { Face Shield }\end{array}$ & Link & $22-04-2020$ & India & $14-04-2020$ & 1968 & Yes & $\begin{array}{l}\text { https://drdo.gov. } \\
\text { in/counter-covid-19- } \\
\text { technologies }\end{array}$ \\
\hline 63 & DRDE & $\begin{array}{l}\text { Defence } \\
\text { research }\end{array}$ & $\begin{array}{l}\text { Hand } \\
\text { Sanitizer, } \\
\text { Face Masks }\end{array}$ & Link & 09-06-2020 & India & $14-04-2020$ & 1973 & Yes & $\begin{array}{l}\text { https://drdo.gov. } \\
\text { in/counter-covid-19- } \\
\text { technologies }\end{array}$ \\
\hline 64 & $\begin{array}{l}\text { RCI } \\
\text { Hyderabad }\end{array}$ & $\begin{array}{l}\text { Defence } \\
\text { research }\end{array}$ & $\begin{array}{l}\text { Hand } \\
\text { Sanitizer, } \\
\text { Face Shield, } \\
\text { Ventilators, } \\
\text { UVC } \\
\text { Disinfection } \\
\text { Product } \\
\end{array}$ & Link & $09-06-2020$ & India & $14-04-2020$ & 1988 & Yes & $\begin{array}{l}\text { https://drdo.gov. } \\
\text { in/counter-covid-19- } \\
\text { technologies }\end{array}$ \\
\hline 65 & $\begin{array}{l}\text { NMRL, } \\
\text { Ambernath }\end{array}$ & $\begin{array}{l}\text { Defence } \\
\text { research }\end{array}$ & $\begin{array}{l}\text { PPE Seam } \\
\text { Sealant }\end{array}$ & Link & $28-05-2020$ & India & $14-04-2020$ & 1953 & Yes & $\begin{array}{l}\text { https://drdo.gov. } \\
\text { in/counter-covid-19- } \\
\text { technologies }\end{array}$ \\
\hline 66 & NPOL, Kochi & $\begin{array}{l}\text { Defence } \\
\text { research }\end{array}$ & $\begin{array}{l}\text { Paper } \\
\text { Disinfector, } \\
\text { Chemical } \\
\text { Disinfectant, } \\
\text { Mobile } \\
\text { Testing Lab, } \\
\text { UVC } \\
\text { Disinfection } \\
\text { Product }\end{array}$ & Link & $20-04-2020$ & India & $14-04-2020$ & 1952 & Yes & $\begin{array}{l}\text { https://drdo.gov. } \\
\text { in/counter-covid-19- } \\
\text { technologies }\end{array}$ \\
\hline 67 & $R \& D E$ & $\begin{array}{l}\text { Defence } \\
\text { research }\end{array}$ & $\begin{array}{l}\text { Isolation } \\
\text { Shelter }\end{array}$ & Link & $20-04-2020$ & India & $14-04-2020$ & 1962 & Yes & $\begin{array}{l}\text { https://drdo.gov. } \\
\text { in/counter-covid-19- } \\
\text { technologies }\end{array}$ \\
\hline 68 & PO-I, DRDO & $\begin{array}{l}\text { Defence } \\
\text { research }\end{array}$ & Face Shield & Link & $22-04-2020$ & India & $14-04-2020$ & 1986 & Yes & $\begin{array}{l}\text { https://drdo.gov. } \\
\text { in/counter-covid-19- } \\
\text { technologies }\end{array}$ \\
\hline 69 & Immutouch & $\begin{array}{l}\text { Engineering } \\
\text { and Robotics }\end{array}$ & $\begin{array}{l}\text { Vibrating } \\
\text { Bracelet for } \\
\text { Surface } \\
\text { Contact }\end{array}$ & Link & 09-05-2020 & $\mathrm{UAE}$ & $29-04-2020$ & 2012 & Yes & $\begin{array}{l}\text { https://www.cbsnews. } \\
\text { com/news/coronavirus- } \\
\text { pandemic-innovations- } \\
\text { hygienehook- } \\
\text { immutouch-smart- } \\
\text { helmet/ }\end{array}$ \\
\hline 70 & DDB ltd & Media Agency & $\begin{array}{l}\text { Hook for } \\
\text { Surface } \\
\text { Contact }\end{array}$ & Link & $02-04-2020$ & England & 29-04-2020 & 1980 & Yes & $\begin{array}{l}\text { https://www.cbsnews. } \\
\text { com/news/coronavirus- } \\
\text { pandemic-innovations- } \\
\text { hygienehook- } \\
\text { immutouch-smart- } \\
\text { helmet/ }\end{array}$ \\
\hline 71 & $\begin{array}{l}\text { Hanwang } \\
\text { Technology } \\
\text { Ltd }\end{array}$ & $\begin{array}{l}\text { Engineering } \\
\text { and Robotics }\end{array}$ & $\begin{array}{l}\text { Smart } \\
\text { Helmet to } \\
\text { detect } \\
\text { temperature }\end{array}$ & Link & $17-05-2020$ & China & $17-03-2020$ & 1998 & No Data & $\begin{array}{l}\text { https://timesofindia. } \\
\text { indiatimes.com/gadgets- } \\
\text { news/watch-police-in- } \\
\text { china-use-smart-helmet- } \\
\text { to-detect-possible- } \\
\text { coronavirus-patients/ } \\
\text { articleshow/74668591.cms }\end{array}$ \\
\hline 72 & Hmcomm & $\begin{array}{l}\text { Engineering } \\
\text { and Robotics }\end{array}$ & $\begin{array}{l}\text { AI - Auto } \\
\text { response } \\
\text { system }\end{array}$ & Link & $25-03-2020$ & Japan & $14-04-2020$ & 2012 & No Data & $\begin{array}{l}\text { https://www. } \\
\text { plugandplaytechcenter. } \\
\text { com/resources/covid-19- } \\
\text { japan-startups- excelling- } \\
\text { time-crisis/ }\end{array}$ \\
\hline 73 & $\begin{array}{l}\text { Mira } \\
\text { Roboticss }\end{array}$ & $\begin{array}{l}\text { Engineering } \\
\text { and Robotics }\end{array}$ & $\begin{array}{l}\text { Robot to } \\
\text { replace } \\
\text { human } \\
\text { workforce }\end{array}$ & Link & $25-03-2020$ & Japan & $14-04-2020$ & 2011 & No Data & $\begin{array}{l}\text { https://www. } \\
\text { plugandplaytechcenter. } \\
\text { com/resources/covid-19- } \\
\text { japan-startups- excelling- } \\
\text { time-crisis/ }\end{array}$ \\
\hline
\end{tabular}

Digital Government: Research and Practice, Vol. 2, No. 2, Article 17. Publication date: December 2020. 


\begin{tabular}{|c|c|c|c|c|c|c|c|c|c|c|}
\hline 74 & Holoeyes & $\begin{array}{l}\text { Engineering } \\
\text { and Robotics }\end{array}$ & $\begin{array}{l}\text { VR Remote } \\
\text { Check-up }\end{array}$ & Link & 25-03-2020 & Japan & $14-04-2020$ & 2016 & No Data & $\begin{array}{l}\text { https://www. } \\
\text { plugandplaytechcenter. } \\
\text { com/resources/covid-19- } \\
\text { japan-startups-excelling- } \\
\text { time-crisis/ }\end{array}$ \\
\hline 75 & Allganize & $\begin{array}{l}\text { Engineering } \\
\text { and Robotics }\end{array}$ & Chatbot App & Link & $25-03-2020$ & Japan & $14-04-2020$ & 2017 & No Data & $\begin{array}{l}\text { https://www. } \\
\text { plugandplaytechcenter. } \\
\text { com/resources/covid-19- } \\
\text { japan-startups-excelling- } \\
\text { time-crisis/ }\end{array}$ \\
\hline 76 & Unirobot & $\begin{array}{l}\text { Engineering } \\
\text { and Robotics }\end{array}$ & $\begin{array}{l}\text { AI based } \\
\text { robots for } \\
\text { home } \\
\text { schooling }\end{array}$ & Link & $25-03-2020$ & Japan & $14-04-2020$ & 2014 & No Data & $\begin{array}{l}\text { https://www. } \\
\text { plugandplaytechcenter. } \\
\text { com/resources/covid-19- } \\
\text { japan-startups-excelling- } \\
\text { time-crisis/ }\end{array}$ \\
\hline 77 & Xlocations & $\begin{array}{l}\text { Engineering } \\
\text { and Robotics }\end{array}$ & $\begin{array}{l}\text { Location AI } \\
\text { platform }\end{array}$ & Link & $25-03-2020$ & Japan & $14-04-2020$ & 2010 & No Data & $\begin{array}{l}\text { https://www. } \\
\text { plugandplaytechcenter. } \\
\text { com/resources/covid-19- } \\
\text { japan-startups-excelling- } \\
\text { time-crisis/ }\end{array}$ \\
\hline 78 & $\begin{array}{l}\text { Asimov } \\
\text { Robotics }\end{array}$ & $\begin{array}{l}\text { Engineering } \\
\text { and Robotics }\end{array}$ & $\begin{array}{l}\text { Medicine } \\
\text { Dispenser, } \\
\text { Trash } \\
\text { Collection, } \\
\text { Disinfection }\end{array}$ & Link & 06-05-2020 & India & $\begin{array}{c}\text { Not } \\
\text { mentioned }\end{array}$ & 2000 & No Data & $\begin{array}{l}\text { https://timesofindia. } \\
\text { indiatimes.com/city/ } \\
\text { kochi/meet-keralas-robo- } \\
\text { warriors-battling-covid- } \\
\text { 19/articleshow/75527447. } \\
\text { cms }\end{array}$ \\
\hline 79 & $\begin{array}{l}\text { Bysh } \\
\text { Automations }\end{array}$ & $\begin{array}{l}\text { Home } \\
\text { Automation }\end{array}$ & $\begin{array}{l}\text { Hand } \\
\text { Sanitizer } \\
\text { robot }\end{array}$ & Link & 06-05-2020 & India & $\begin{array}{c}\text { Not } \\
\text { mentioned }\end{array}$ & 2013 & No Data & $\begin{array}{l}\text { https://timesofindia. } \\
\text { indiatimes.com/city/ } \\
\text { kochi/meet-keralas-robo- } \\
\text { warriors-battling-covid- } \\
\text { 19/articleshow/75527447. } \\
\text { cms }\end{array}$ \\
\hline 80 & $\begin{array}{l}\text { Dhee Yantra } \\
\text { Research labs }\end{array}$ & $\begin{array}{l}\text { Engineering } \\
\text { and Robotics }\end{array}$ & \begin{tabular}{|l|} 
App \\
providing \\
Information
\end{tabular} & Link & $05-04-2020$ & India & 05-04-2020 & 2017 & Yes & $\begin{array}{l}\text { https://www. } \\
\text { deccanherald.com/ } \\
\text { business/technology/ } \\
\text { kerala-technologys- } \\
\text { helping-hands-at-the- } \\
\text { time- of-covid-19-821607. } \\
\text { html }\end{array}$ \\
\hline 81 & Bluesemi & $\begin{array}{l}\text { Engineering } \\
\text { and Robotics }\end{array}$ & $\begin{array}{l}\text { Thermal } \\
\text { sensor }\end{array}$ & Link & 04-05-2020 & India & 04-05-2020 & 2017 & Yes & $\begin{array}{l}\text { https://www. } \\
\text { financialexpress.com// } \\
\text { industry/technology// } \\
\text { disruption-hub-9- } \\
\text { startups-at-hyderabad- } \\
\text { based-startup-incubator- } \\
\text { t-hub-debut-niche- } \\
\text { solutions-to-combat- } \\
\text { covid-19/1946910/ }\end{array}$ \\
\hline 82 & Byteforce & $\begin{array}{l}\text { Engineering } \\
\text { and Robotics }\end{array}$ & $\begin{array}{l}\text { AI-based } \\
\text { People } \\
\text { Monitoring } \\
\text { App }\end{array}$ & Link & 04-05-2020 & India & 04-05-2020 & 2017 & Yes & $\begin{array}{l}\text { https://www. } \\
\text { financialexpress.com/ } \\
\text { industry/technology/ } \\
\text { disruption-hub-9- } \\
\text { startups-at-hyderabad- } \\
\text { based-startup-incubator- } \\
\text { t-hub-debut-nich-- } \\
\text { solutions-to-combat- } \\
\text { covid-19/1946910/ }\end{array}$ \\
\hline
\end{tabular}

Digital Government: Research and Practice, Vol. 2, No. 2, Article 17. Publication date: December 2020. 
Global Pandemic and Rapid New Product Development of Medical Products

\begin{tabular}{|c|c|c|c|c|c|c|c|c|c|c|}
\hline 83 & Blocksapp.ai & $\begin{array}{l}\text { Engineering } \\
\text { and Robotics }\end{array}$ & $\begin{array}{l}\text { AI-based } \\
\text { drone } \\
\text { monitoring } \\
\text { solution }\end{array}$ & Link & 04-05-2020 & India & 04-05-2020 & 2019 & Yes & $\begin{array}{l}\text { https://www. } \\
\text { financialexpress.com/ } \\
\text { industry/technology/ } \\
\text { disruption-hub-9- } \\
\text { startups-at-hyderabad- } \\
\text { based-startup-incubator- } \\
\text { t-hub-debut-niche- } \\
\text { solutions-to-combat- } \\
\text { covid-19/1946910/ }\end{array}$ \\
\hline 84 & Cogni.Care & $\begin{array}{l}\text { Engineering } \\
\text { and Robotics }\end{array}$ & $\begin{array}{l}\text { Thermal } \\
\text { sensor, } \\
\text { Ventilators }\end{array}$ & Link & 04-05-2020 & India & 04-05-2020 & 1994 & Yes & $\begin{array}{l}\text { https://www. } \\
\text { financialexpress.com/ } \\
\text { industry/technology/ } \\
\text { disruption-hub-9- } \\
\text { startups-at-hyderabad- } \\
\text { based-startup-incubator- } \\
\text { t-hub-debut-niche- } \\
\text { solutions-to-combat- } \\
\text { covid-19/1946910/ }\end{array}$ \\
\hline 85 & $\begin{array}{l}\text { Dimension } \\
\text { NXG }\end{array}$ & $\begin{array}{l}\text { Engineering } \\
\text { and Robotics }\end{array}$ & $\begin{array}{l}\text { Large-scale } \\
\text { thermal } \\
\text { scanning } \\
\text { glasses }\end{array}$ & Link & 04-05-2020 & India & 04-05-2020 & 2014 & Yes & $\begin{array}{l}\text { https://www. } \\
\text { financialexpress.com/ } \\
\text { industry/technology/ } \\
\text { disruption-hub-9- } \\
\text { startups-at-hyderabad- } \\
\text { based-startup-incubator- } \\
\text { t-hub-debut-niche- } \\
\text { solutions-to-combat- } \\
\text { covid-19/1946910/ }\end{array}$ \\
\hline 86 & Marut & $\begin{array}{l}\text { Engineering } \\
\text { and Robotics }\end{array}$ & $\begin{array}{l}\text { Drone based } \\
\text { chemical } \\
\text { disinfectant }\end{array}$ & Link & 04-05-2020 & India & 04-05-2020 & 2019 & Yes & $\begin{array}{l}\text { https://www. } \\
\text { financialexpress.com/ } \\
\text { industry/technology/ } \\
\text { disruption-hub-9- } \\
\text { startups-at-hyderabad- } \\
\text { based-startup-incubator- } \\
\text { t-hub-debut-niche- } \\
\text { solutions-to-combat- } \\
\text { covid-19/1946910/ }\end{array}$ \\
\hline 87 & Tericsoft & $\begin{array}{l}\text { Engineering } \\
\text { and Robotics }\end{array}$ & $\begin{array}{l}\text { AI-based } \\
\text { People } \\
\text { Monitoring } \\
\text { App }\end{array}$ & Link & 04-05-2020 & India & 04-05-2020 & 2018 & Yes & $\begin{array}{l}\text { https://www. } \\
\text { financialexpress.com/ } \\
\text { industry/technology/ } \\
\text { disruption-hub-9- } \\
\text { startups-at-hyderabad- } \\
\text { based-startup-incubator- } \\
\text { t-hub-debut-niche- } \\
\text { solutions-to-combat- } \\
\text { covid-19/1946910/ }\end{array}$ \\
\hline 88 & $\begin{array}{l}\text { Eyestem } \\
\text { Research }\end{array}$ & $\begin{array}{l}\text { Biotechnology } \\
\text { Company }\end{array}$ & $\begin{array}{l}\text { Drug } \\
\text { screening } \\
\text { platform }\end{array}$ & Link & $25-04-2020$ & India & $\begin{array}{c}\text { Not } \\
\text { mentioned }\end{array}$ & 2015 & No Data & $\begin{array}{l}\text { https://timesofindia. } \\
\text { indiatimes.com/india/ } \\
\text { covid-19-over-1000-tech- } \\
\text { proposals-in-4-weeks- } \\
\text { by-start-ups/articleshow/ } \\
\text { 75377701.cms }\end{array}$ \\
\hline 89 & Kerasiev & $\begin{array}{l}\text { Chemical } \\
\text { Industry }\end{array}$ & $\begin{array}{l}\text { Wastewater } \\
\text { filtation } \\
\text { plant }\end{array}$ & Link & $25-04-2020$ & India & $\begin{array}{c}\text { Not } \\
\text { mentioned }\end{array}$ & 2017 & No Data & $\begin{array}{l}\text { https://timesofindia. } \\
\text { indiatimes.com/india/ } \\
\text { covid-19-over-1000-tech- } \\
\text { proposals-in-4-weeks- } \\
\text { by-start-ups/articleshow/ } \\
\text { 75377701.cms }\end{array}$ \\
\hline 90 & Innaumation & $\begin{array}{l}\text { Medical } \\
\text { equipment }\end{array}$ & $\begin{array}{l}\text { Convascelent } \\
\text { plasma } \\
\text { therapy }\end{array}$ & Link & $25-04-2020$ & India & $25-04-2020$ & 2017 & Yes & $\begin{array}{l}\text { https://timesofindia. } \\
\text { indiatimes.com/india/ } \\
\text { covid-19-over-1000-tech- } \\
\text { proposals-in-4-weeks- } \\
\text { by-start-ups/articleshow/ } \\
\text { 75377701.cms }\end{array}$ \\
\hline
\end{tabular}

Digital Government: Research and Practice, Vol. 2, No. 2, Article 17. Publication date: December 2020. 
17:20 - M. C. Kumari and B. Sagar

\begin{tabular}{|c|c|c|c|c|c|c|c|c|c|c|}
\hline 91 & $\begin{array}{l}\text { Pluss } \\
\text { technologies }\end{array}$ & $\begin{array}{l}\text { Software } \\
\text { Company }\end{array}$ & $\begin{array}{l}\text { Cold Chain } \\
\text { for Swabs }\end{array}$ & Link & $25-04-2020$ & India & $25-04-2020$ & 2016 & Yes & $\begin{array}{l}\text { https://timesofindia. } \\
\text { indiatimes.com/india/ } \\
\text { covid-19-over-1000-tech- } \\
\text { proposals-in-4-weeks- } \\
\text { by-start-ups/articleshow/ } \\
\text { 75377701.cms }\end{array}$ \\
\hline 92 & DNAXperts & $\begin{array}{l}\text { Biotechnology } \\
\text { Company }\end{array}$ & $\begin{array}{l}\text { Real time } \\
\text { PCR kit }\end{array}$ & Link & $25-04-2020$ & India & $25-04-2020$ & 2015 & Yes & $\begin{array}{l}\text { https://timesofindia. } \\
\text { indiatimes.com/india/ } \\
\text { covid-19-over-1000-tech- } \\
\text { proposals-in-4-weeks- } \\
\text { by-start-ups/articleshow/ } \\
\text { 75377701.cms }\end{array}$ \\
\hline 93 & Docturnal & $\begin{array}{l}\text { Biotechnology } \\
\text { Company }\end{array}$ & $\begin{array}{l}\text { Repurposed } \\
\text { TB screening } \\
\text { app }\end{array}$ & Link & $17-05-2020$ & India & $17-05-2020$ & 2016 & Yes & $\begin{array}{l}\text { https://yourstory.com/ } \\
\text { 2020/05/medtech-startup- } \\
\text { docturnal-coronavirus- } \\
\text { covid-19-screening }\end{array}$ \\
\hline 94 & Stempeutics & $\begin{array}{l}\text { Biotechnology } \\
\text { Company }\end{array}$ & $\begin{array}{l}\text { Approach in } \\
\text { therapeutics }\end{array}$ & Link & $18-04-2020$ & India & $18-04-2020$ & 2006 & Yes & $\begin{array}{l}\text { https://www. } \\
\text { thehindubusinessline. } \\
\text { com/news/science/c- } \\
\text { camps-covid-19- } \\
\text { accelerator-shortlists- 7- } \\
\text { deep-science- } \\
\text { innovations-to-fight- } \\
\text { virus/article31373999.ece }\end{array}$ \\
\hline 95 & $\begin{array}{l}\text { Huwel Life } \\
\text { Sciences }\end{array}$ & $\begin{array}{l}\text { Biotechnology } \\
\text { Company }\end{array}$ & $\begin{array}{l}\text { Rapid } \\
\text { Diagnostics }\end{array}$ & Link & $18-04-2020$ & China & $18-04-2020$ & 2015 & No Data & $\begin{array}{l}\text { https://www. } \\
\text { thehindubusinessline. } \\
\text { com/news/science/c- } \\
\text { camps-covid-19- } \\
\text { accelerator-shortlists-7- } \\
\text { deep-science- } \\
\text { innovations-to-fight- } \\
\text { virus/article31373999.ece }\end{array}$ \\
\hline 96 & $\begin{array}{l}\text { Ubiqare } \\
\text { Health }\end{array}$ & $\begin{array}{l}\text { Biotechnology } \\
\text { Company }\end{array}$ & $\begin{array}{l}\text { Telemedicine } \\
\text { support }\end{array}$ & Link & $18-04-2020$ & India & $18-04-2020$ & 2017 & Yes & $\begin{array}{l}\text { https://www. } \\
\text { thehindubusinessline. } \\
\text { com/news/science/c- } \\
\text { camps-covid-19- } \\
\text { accelerator-shortlists-7- } \\
\text { deep-science- } \\
\text { innovations-to-fight- } \\
\text { virus/article31373999.ece }\end{array}$ \\
\hline 97 & Magna & $\begin{array}{l}\text { Consumer } \\
\text { Goods }\end{array}$ & Face Masks & Link & $20-04-2020$ & Canada & 01-04-2020 & 1957 & No Data & $\begin{array}{l}\text { https://www.magna.com/ } \\
\text { coronavirus/magna- } \\
\text { employees-in-action }\end{array}$ \\
\hline 98 & $\begin{array}{l}\text { General } \\
\text { Motors }\end{array}$ & Automotive & $\begin{array}{l}\text { PPE, } \\
\text { Ventilators }\end{array}$ & Link & $27-04-2020$ & USA & $27-04-2020$ & 1908 & Yes & $\begin{array}{l}\text { https://www.cbsnews. } \\
\text { com/news/ford-general- } \\
\text { motors-coronavirus- } \\
\text { ventilators-medical- } \\
\text { supplies/ }\end{array}$ \\
\hline 99 & \begin{tabular}{|l} 
ABC \\
Technology
\end{tabular} & $\begin{array}{l}\text { Consumer } \\
\text { Goods }\end{array}$ & $\begin{array}{l}\text { Ventilators, } \\
\text { Face Shield, } \\
\text { Face Mask }\end{array}$ & Link & 03-04-2020 & Canada & 03-04-2020 & 2013 & No Data & $\begin{array}{l}\text { https://abctechnologies. } \\
\text { com/wp-content/uploads/ } \\
\text { ABCTechnologies_- } \\
\text { MedicalDevicesUnit_ } \\
\text { PressRelease_Final1-1.pdf }\end{array}$ \\
\hline 100 & Linamar & Automotive & Ventilators & Link & $02-05-2020$ & Canada & 01-04-2020 & 1966 & No Data & $\begin{array}{l}\text { https://www. } \\
\text { linamar.com/coronavirus/ } \\
\text { community }\end{array}$ \\
\hline 101 & Ford & Automotive & Respirators & Link & $06-05-2020$ & USA & 01-04-2020 & 1903 & Yes & $\begin{array}{l}\text { https://corporate. } \\
\text { ford.com/articles/ } \\
\text { products/ford-and-3m- } \\
\text { now-shipping-powered- } \\
\text { air-purifying-respirators. } \\
\text { html }\end{array}$ \\
\hline
\end{tabular}

Digital Government: Research and Practice, Vol. 2, No. 2, Article 17. Publication date: December 2020. 


\begin{tabular}{|c|c|c|c|c|c|c|c|c|c|c|}
\hline 102 & $3 \mathrm{M}$ & Conglomerate & PPE Kits & Link & $11-05-2020$ & USA & 01-04-2020 & 1902 & Yes & $\begin{array}{l}\text { https://www.3m.com/3M/ } \\
\text { en_US/worker-health- } \\
\text { safety-us/covid19/ } \\
\text { \#general-respiratory }\end{array}$ \\
\hline 103 & Ferrari & Automotive & $\begin{array}{l}\text { Respirator } \\
\text { valves and } \\
\text { fittings }\end{array}$ & Link & $16-04-2020$ & Italy & $\begin{array}{c}\text { Not } \\
\text { mentioned }\end{array}$ & 1947 & No Data & $\begin{array}{l}\text { https://corporate.ferrari. } \\
\text { com/en/ferrari-continues- } \\
\text { its-efforts- fight-covid-19- } \\
\text { pandemic }\end{array}$ \\
\hline 104 & Lamborghini & Automotive & $\begin{array}{l}\text { Surgical } \\
\text { Masks, Face } \\
\text { Shields }\end{array}$ & Link & $31-03-2020$ & Italy & $31-04-2020$ & 1963 & Yes & $\begin{array}{l}\text { https://www.lamborghini. } \\
\text { com/en-en/news/covid-19- } \\
\text { lamborghini-is-producing- } \\
\text { surgical-masks-and- } \\
\text { medical-shields }\end{array}$ \\
\hline 105 & $\begin{array}{l}\text { Maruti } \\
\text { Suzuki }\end{array}$ & Automotive & $\begin{array}{l}\text { PPE Kits, } \\
\text { Ventilators }\end{array}$ & Link & $28-04-2020$ & India & $28-04-2020$ & 1982 & Yes & $\begin{array}{l}\text { https://www. } \\
\text { marutisuzuki.com/ } \\
\text { corporate/media/press- } \\
\text { releases/2020/maruti- } \\
\text { suzuki-mobilizes- } \\
\text { production-of-ventilators }\end{array}$ \\
\hline 106 & $\begin{array}{l}\text { Hero } \\
\text { MotoCorp }\end{array}$ & Automotive & $\begin{array}{l}\text { Hand } \\
\text { Sanitizers }\end{array}$ & Link & $18-05-2020$ & India & $18-05-2020$ & 1984 & Yes & $\begin{array}{l}\text { https://www. } \\
\text { financialexpress.com/auto/ } \\
\text { industry/covid-19- } \\
\text { coronavirus-relief-hero- } \\
\text { motocorp-manufactures- } \\
\text { over-5000-litre-sanitiser- } \\
\text { to-fight-the-pandemic- } \\
\text { ration-kits-food-supplies- } \\
\text { donations/1933714/ }\end{array}$ \\
\hline 107 & Reebok & $\begin{array}{l}\text { Retail } \\
\text { Company }\end{array}$ & PPE Kits & Link & $14-04-2020$ & USA & 01-04-2020 & 1958 & Yes & $\begin{array}{l}\text { https://www.reebok.com/ } \\
\text { us/giveback }\end{array}$ \\
\hline 108 & Nike & $\begin{array}{l}\text { Retail } \\
\text { Company }\end{array}$ & $\begin{array}{l}\text { Shoes for } \\
\text { healthcare } \\
\text { workers; } \\
\text { T-shirts } \\
\text { under PPEs }\end{array}$ & Link & 04-05-2020 & USA & $27-05-2020$ & 1964 & Yes & $\begin{array}{l}\text { https://purpose.nike.com/ } \\
\text { covid-19-response-efforts }\end{array}$ \\
\hline 109 & Honda & Automotive & $\begin{array}{l}\text { Ventilator } \\
\text { component, } \\
\text { Face Shields }\end{array}$ & Link & 05-05-2020 & Japan & $05-05-2020$ & 1948 & No Data & $\begin{array}{l}\text { https:/hondanews.com/en- } \\
\text { US/honda-corporate/ } \\
\text { releases/release- } \\
\text { b262241b363797081e713bf59 } \\
\text { 9003a5a-detroit-area- } \\
\text { residents-will-be- } \\
\text { transported-to-covid-19- } \\
\text { testing-in-modified-honda- } \\
\text { odyssey-minivans } \\
\end{array}$ \\
\hline 110 & Volkswagen & Automotive & Ventilators & Link & $30-03-2020$ & Germany & $\begin{array}{c}\text { Not } \\
\text { mentioned }\end{array}$ & 1937 & No Data & $\begin{array}{l}\text { https://www. } \\
\text { volkswagenag.com/en/ } \\
\text { news/2020/03/ } \\
\text { cars_to_ventilators.html }\end{array}$ \\
\hline 111 & $\begin{array}{l}\text { Mercedes } \\
\text { Benz }\end{array}$ & Automotive & $\begin{array}{l}\text { Medical } \\
\text { equipments, } \\
\text { Face shield }\end{array}$ & Link & $15-04-2020$ & Germany & 01-04-2020 & 1926 & Yes & $\begin{array}{l}\text { https://www.daimler.com/ } \\
\text { sustainability/corporate- } \\
\text { citizenship/the-covid-19- } \\
\text { pandemic-daimler-is- } \\
\text { helping.html }\end{array}$ \\
\hline 112 & $\begin{array}{l}\text { Fiat Chrysler } \\
\text { Automobiles }\end{array}$ & Automotive & Face masks & Link & $27-03-2020$ & England & $\begin{array}{c}\text { Not } \\
\text { mentioned }\end{array}$ & 2014 & No Data & $\begin{array}{l}\text { https://www. } \\
\text { manufacturingglobal.com/ } \\
\text { smart-manufacturing/fiat- } \\
\text { chrysler-automobiles-fca- } \\
\text { response-covid-19 }\end{array}$ \\
\hline 113 & Nissan & Automotive & $\begin{array}{l}\text { Face shields, } \\
\text { Hospital } \\
\text { gowns, } \\
\text { Ventilators, } \\
\text { Face masks }\end{array}$ & Link & $25-05-2020$ & Japan & $25-05-2020$ & 1933 & No Data & $\begin{array}{l}\text { https://global.nissanstories. } \\
\text { com/en/releases/release- } \\
\text { aba6b763b790428810f6fe31a80 } \\
\text { 07e34-global-social- } \\
\text { initiatives-against-covid-19 }\end{array}$ \\
\hline
\end{tabular}

Digital Government: Research and Practice, Vol. 2, No. 2, Article 17. Publication date: December 2020. 


\begin{tabular}{|c|c|c|c|c|c|c|c|c|c|c|}
\hline 114 & Tesla & Automotive & $\begin{array}{l}\text { Ventilators, } \\
\text { Vaccines }\end{array}$ & Link & 03-07-2020 & USA & 03-07-2020 & 2003 & No & $\begin{array}{l}\text { https://timesofindia. } \\
\text { indiatimes.com/gadgets- } \\
\text { news//elon-musks-tesla- } \\
\text { to-help-pharma- } \\
\text { company-in- } \\
\text { development- } \\
\text { of-covid-19-vaccine/ } \\
\text { articleshow/76762165.cms }\end{array}$ \\
\hline 115 & Volvo & Automotive & $\begin{array}{l}\text { Face Shields, } \\
\text { Face Masks }\end{array}$ & Link & 09-04-2020 & Sweden & 01-04-2020 & 1927 & No Data & $\begin{array}{l}\text { https://www.volvogroup. } \\
\text { com/en-en/covid-19.html }\end{array}$ \\
\hline 116 & Renault & Automotive & $\begin{array}{l}\text { Respirators, } \\
\text { Face Masks, } \\
\text { Face Shield }\end{array}$ & Link & $31-03-2020$ & France & $31-03-2020$ & 1898 & Yes & $\begin{array}{l}\text { https://group.renault. } \\
\text { com/en/news-on-air/ } \\
\text { news/groupe-renaults- } \\
\text { initiatives-to-help- } \\
\text { tackle-the-covid-19- } \\
\text { crisis/ }\end{array}$ \\
\hline 117 & Groupe PSA & Automotive & $\begin{array}{l}\text { Respirators, } \\
\text { Face Masks, } \\
\text { Face Shield }\end{array}$ & Link & $16-04-2020$ & France & 01-04-2020 & 1976 & Yes & $\begin{array}{l}\text { https://www.groupe-psa. } \\
\text { com/en/newsroom/ } \\
\text { corporate-en/covid-19- } \\
\text { crisis- groupe-psa-is- } \\
\text { mobilizing/ }\end{array}$ \\
\hline 118 & Lexus & Automotive & $\begin{array}{l}\text { Ventilators, } \\
\text { Face Masks }\end{array}$ & Link & 20-04-2020 & Japan & 01-04-2020 & 1989 & No Data & $\begin{array}{l}\text { http://media.lexus.ca/ } \\
\text { releases/supporting- } \\
\text { canadian-communities- } \\
\text { our-covid-19-response }\end{array}$ \\
\hline 119 & Cadillac & Automotive & PPE Kits & Link & $14-04-2020$ & USA & $14-04-2020$ & 1902 & Yes & \begin{tabular}{|l} 
https://www. \\
globenewswire.com/ \\
news-release/2020/04/14/ \\
2015928/0/en/Cadillac- \\
Products-Manufacturing- \\
Medical-PPE-to-Help- \\
Healthcare-Workers- \\
Fight-COVID-19-in- \\
Michigan.html
\end{tabular} \\
\hline 120 & Wildcraft & \begin{tabular}{|l} 
Retail \\
Company
\end{tabular} & $\begin{array}{l}\text { PPE Kits, } \\
\text { Face Masks }\end{array}$ & Link & $11-06-2020$ & India & $\begin{array}{c}\text { Not } \\
\text { mentioned }\end{array}$ & 1998 & No Data & $\begin{array}{l}\text { https://www.thehindu. } \\
\text { com/business/wildcraft- } \\
\text { unveils-covid-19-gear- } \\
\text { ramps-up-staff/ } \\
\text { article31807291.ece }\end{array}$ \\
\hline 121 & Mazda & Automotive & Face Shields & Link & $26-05-2020$ & Japan & $\begin{array}{c}\text { Not } \\
\text { mentioned }\end{array}$ & 1920 & No Data & $\begin{array}{l}\text { https://newsroom. } \\
\text { mazda.com/assets/ } \\
\text { 100187/200526_ENG__ } \\
\text { Information.pdf?_ga=2. } \\
\text { 105919747.1496763394. } \\
\text { 1594455048-975523656. } \\
\text { 1594455048 }\end{array}$ \\
\hline 122 & Skoda & Automotive & Respirators & Link & $30-04-2020$ & Czechia & $30-04-2020$ & 1895 & No & $\begin{array}{l}\text { https://www.skoda- } \\
\text { storyboard.com/en/press- } \\
\text { releases/skoda-autos- } \\
\text { support-in-fighting- } \\
\text { covid-19-met-with-high- } \\
\text { demand/ }\end{array}$ \\
\hline 123 & BHEL & $\begin{array}{l}\text { Consumer } \\
\text { appliances }\end{array}$ & $\begin{array}{l}\text { Hand } \\
\text { Sanitizers }\end{array}$ & Link & 30-03-2020 & India & $30-03-2020$ & 1964 & Yes & $\begin{array}{l}\text { http://www.bhel.com/ } \\
\text { assets/downloads/ } \\
\text { 5e81a823af6dfBHEL_and_ } \\
\text { its_employees_- } \\
\text { contribute_- } \\
\text { wholeheartedly_lead_ } \\
\text { the_way_in_helping_the_ } \\
\text { nation_in_its_fight_ } \\
\text { against_Covid-19.pdf }\end{array}$ \\
\hline
\end{tabular}

Digital Government: Research and Practice, Vol. 2, No. 2, Article 17. Publication date: December 2020. 


\begin{tabular}{|c|c|c|c|c|c|c|c|c|c|c|}
\hline 124 & Unilever & \begin{tabular}{|l} 
Consumer \\
goods
\end{tabular} & $\begin{array}{l}\text { Ventilator, } \\
\text { Chemical } \\
\text { Disinfectant }\end{array}$ & Link & 25-04-2020 & India & 01-04-2020 & 1929 & Yes & $\begin{array}{l}\text { https://www.unilever.com/ } \\
\text { news/covid-response/ }\end{array}$ \\
\hline 125 & Bosch & Automotive & \begin{tabular}{|l} 
Diagnostic \\
test tool
\end{tabular} & Link & 26-03-2020 & Germany & $\begin{array}{c}\text { Not } \\
\text { mentioned }\end{array}$ & 1886 & No Data & $\begin{array}{l}\text { https://www.news18.com/ } \\
\text { news/world/bosch- } \\
\text { develops-covid-19-test- } \\
\text { tool-to-detect-coronavirus- } \\
\text { in-under-3-hours-2552481. } \\
\text { html }\end{array}$ \\
\hline 126 & SC Johnson & $\begin{array}{l}\text { Consumer } \\
\text { goods }\end{array}$ & $\begin{array}{l}\text { Hand } \\
\text { Sanitizer }\end{array}$ & Link & 22-04-2020 & USA & $\begin{array}{c}\text { Not } \\
\text { mentioned }\end{array}$ & 1886 & No Data & $\begin{array}{l}\text { https://www.3blmedia.com/ } \\
\text { News/SC-Johnson- } \\
\text { Converts-Line-Largest- } \\
\text { Global-Manufacturing- } \\
\text { Plant-Produce-Hand- } \\
\text { Sanitizer-COVID-19 } \\
\end{array}$ \\
\hline 127 & Redbull & Brewing & Respirators & Link & $14-3-2020$ & Austria & 01-04-2020 & 1984 & Yes & $\begin{array}{l}\text { https://motorsport. } \\
\text { tech/formula-1/red-bull- } \\
\text { racing-keeping-busy-in- } \\
\text { fight-against-coronavirus- } \\
\text { pandemic }\end{array}$ \\
\hline 128 & Nordstrom & \begin{tabular}{|l|} 
Retail \\
Company
\end{tabular} & Face Masks & Link & 20-03-2020 & USA & 01-04-2020 & 1901 & Yes & \begin{tabular}{|l|} 
https://press.nordstrom. \\
com/news-releases/news- \\
release-details/working- \\
towards-nearly-1-million- \\
masks \\
\end{tabular} \\
\hline 129 & Honeywell & Aerospace & $\begin{array}{l}\text { Hand } \\
\text { Sanitizer }\end{array}$ & Link & 02-04-2020 & USA & 01-04-2020 & 1906 & Yes & $\begin{array}{l}\text { https://www.honeywell. } \\
\text { com/us/en/news/2020/04/ } \\
\text { how-honeywell-plants- } \\
\text { pivoted-to-hand-sanitizer }\end{array}$ \\
\hline 130 & $\begin{array}{l}\text { Mahindra } \\
\text { and } \\
\text { Mahindra }\end{array}$ & Automotive & \begin{tabular}{|l} 
Respirators, \\
Face Masks, \\
Face Shields, \\
Ventilators, \\
Hand \\
Sanitisers \\
\end{tabular} & Link & 21-04-2020 & India & 01-04-2020 & 1945 & Yes & $\begin{array}{l}\text { https://www.mahindra. } \\
\text { com/news-room/ } \\
\text { knowledge-centre/impact- } \\
\text { stories/mahindra-rising-to- } \\
\text { support-the-nation-in-the- } \\
\text { fight-against-covid-19 } \\
\end{array}$ \\
\hline 131 & $\begin{array}{l}\text { Brooks } \\
\text { Brothers }\end{array}$ & $\begin{array}{l}\text { Retail } \\
\text { Company }\end{array}$ & \begin{tabular}{|l} 
Surgical \\
Masks, \\
Hospital \\
gowns
\end{tabular} & Link & 28-04-2020 & USA & 28-04-2020 & 1818 & Yes & $\begin{array}{l}\text { https://www.triplepundit. } \\
\text { com/story/2020/ceos- } \\
\text { respond-coronavirus- } \\
\text { covid-19/87361 }\end{array}$ \\
\hline 132 & Bacardi & Brewing & \begin{tabular}{|l} 
Hand \\
Sanitizer
\end{tabular} & Link & 25-03-2020 & Bermuda & 25-03-2020 & 1862 & No & $\begin{array}{l}\text { https://www.3blmedia.com/ } \\
\text { News/USA-France-and- } \\
\text { UK-Mexico-Bacardi- } \\
\text { Diverts-Global-Production- } \\
\text { Increase-Hand-Sanitizer- } \\
\text { Supply-Local }\end{array}$ \\
\hline 133 & Hanesbrands & $\begin{array}{l}\text { Retail } \\
\text { Company }\end{array}$ & $\begin{array}{l}\text { Ventilators, } \\
\text { Emergency } \\
\text { gear }\end{array}$ & Link & $30-03-2020$ & USA & $30-03-2020$ & 2006 & Yes & $\begin{array}{l}\text { https://www.fastcompany. } \\
\text { com/90483109/inside-the- } \\
\text { mad-rush-to-produce- } \\
\text { ventilators-and- } \\
\text { emergency-gear-to-fight- } \\
\text { covid-19 }\end{array}$ \\
\hline 134 & H\&M & $\begin{array}{l}\text { Retail } \\
\text { Company }\end{array}$ & PPE Kits & Link & 22-03-2020 & Sweden & 22-03-2020 & 1947 & No Data & $\begin{array}{l}\text { https://www.ecotextile. } \\
\text { com/2020032225865/ } \\
\text { fashion-retail-news/h-m- } \\
\text { suppliers-to-make-ppe-to- } \\
\text { tackle-covid-19.html }\end{array}$ \\
\hline 135 & Reformation & $\begin{array}{l}\text { Retail } \\
\text { Company }\end{array}$ & Face Masks & Link & $13-04-2020$ & USA & 13-04-2020 & 2009 & Yes & $\begin{array}{l}\text { https://nrf.com/ } \\
\text { blog/retailers-lead-mask- } \\
\text { production-combat-covid- } \\
19\end{array}$ \\
\hline
\end{tabular}

Digital Government: Research and Practice, Vol. 2, No. 2, Article 17. Publication date: December 2020. 


\begin{tabular}{|c|c|c|c|c|c|c|c|c|c|c|}
\hline 136 & Nestle & $\begin{array}{l}\text { Food } \\
\text { Processing }\end{array}$ & Ventilators & Link & $25-05-2020$ & Switzerland & $25-05-2020$ & 1866 & No Data & $\begin{array}{l}\text { https://www.nestle.com/ } \\
\text { media/news/nestle-cote- } \\
\text { ivoire-donates-ventilators- } \\
\text { covid-19 }\end{array}$ \\
\hline 137 & $\begin{array}{l}\text { Brown } \\
\text { Forman }\end{array}$ & Brewing & $\begin{array}{l}\text { Hand } \\
\text { Sanitizer }\end{array}$ & Link & 23-04-2020 & USA & 23-04-2020 & 1870 & Yes & $\begin{array}{l}\text { https://www.3blmedia.com/ } \\
\text { News/Brown-Forman- } \\
\text { Produces-Hand-Sanitizer- } \\
\text { Employees-Health-Care- } \\
\text { Providers-and-First- } \\
\text { Responders }\end{array}$ \\
\hline 138 & IKEA & $\begin{array}{l}\text { Retail } \\
\text { Company }\end{array}$ & Face Masks & Link & $31-03-2020$ & India & 31-03-2020 & 1943 & Yes & $\begin{array}{l}\text { https://www.reuters.com/ } \\
\text { article/us-health- } \\
\text { coronavirus-ikea-supply- } \\
\text { idUSKBN21I1EK?taid= } \\
\text { 5e836a499a7fcd0001c49c63\& } \\
\text { utm_campaign= } \\
\text { trueAnthem\%3A } \pm \\
\text { Trending } \pm \text { Content\& } \\
\text { utm_medium= } \\
\text { trueAnthem\&utm_source= } \\
\text { twitter }\end{array}$ \\
\hline 139 & 58Gin & Brewing & $\begin{array}{l}\text { Hand } \\
\text { Sanitizer }\end{array}$ & Link & $13-04-2020$ & England & 13-04-2020 & 2016 & Yes & $\begin{array}{l}\text { https://www.bbc.com/ } \\
\text { worklife/article/20200413- } \\
\text { how-factories-change- } \\
\text { production-to-quickly- } \\
\text { fight-coronavirus }\end{array}$ \\
\hline 140 & Royal Mint & $\begin{array}{l}\text { Coin and Medal } \\
\text { Production }\end{array}$ & Face Shields & Link & $13-04-2020$ & England & $13-04-2020$ & 886 & Yes & $\begin{array}{l}\text { https://www.bbc.com/ } \\
\text { worklife/article/20200413- } \\
\text { how-factories-change- } \\
\text { production-to-quickly- } \\
\text { fight-coronavirus }\end{array}$ \\
\hline 141 & Sharp & $\begin{array}{l}\text { Consumer } \\
\text { Appliances }\end{array}$ & $\begin{array}{l}\text { Surgical } \\
\text { Mask }\end{array}$ & Link & 02-03-2020 & Japan & $02-03-2020$ & 1912 & No Data & $\begin{array}{l}\text { https://www.bbc. } \\
\text { com/news/technology- } \\
51706629\end{array}$ \\
\hline 142 & SGMW & Automotive & Face Masks & Link & $13-04-2020$ & China & $\begin{array}{c}\text { Not } \\
\text { mentioned }\end{array}$ & 2002 & No Data & $\begin{array}{l}\text { https://www.bbc.com/ } \\
\text { worklife/article/20200413- } \\
\text { how-factories-change- } \\
\text { production-to-quickly- } \\
\text { fight-coronavirus }\end{array}$ \\
\hline 143 & Carbon & $\begin{array}{l}\text { Digital } \\
\text { Manufacturer }\end{array}$ & $\begin{array}{l}\text { Face Shields, } \\
\text { Test Swabs }\end{array}$ & Link & $25-03-2020$ & USA & 01-04-2020 & 2010 & Yes & $\begin{array}{l}\text { https://www.forbes. } \\
\text { com/sites/amyfeldman/ } \\
\text { 2020/03/25/inside-a- } \\
\text { silicon-valley-unicorns- } \\
\text { urgent-dash-to-3d-print- } \\
\text { face-shields-and-test- } \\
\text { swabs-to-battle-covid-19/ } \\
\# 2 b 1281 \text { d14370 }\end{array}$ \\
\hline 144 & $\begin{array}{l}\text { Anheuser- } \\
\text { Busch } \\
\text { InBev }\end{array}$ & Brewing & \begin{tabular}{|l} 
Hand \\
Sanitizer
\end{tabular} & Link & 01-04-2020 & Belgium & 01-04-2020 & 2008 & Yes & $\begin{array}{l}\text { https://www.forbes. } \\
\text { com/sites/giacomotognini/ } \\
\text { 2020/04/01/coronavirus- } \\
\text { business-tracker-how-the- } \\
\text { private-sector-is-fighting- } \\
\text { the- covid-19-pandemic/ } \\
\text { ?sh=60e6572a5899 }\end{array}$ \\
\hline 145 & Aria Designs & Design Studio & $\begin{array}{l}\text { Surgical } \\
\text { Masks }\end{array}$ & Link & 01-04-2020 & India & 01-04-2020 & 2012 & Yes & $\begin{array}{l}\text { https://www.forbes.com/ } \\
\text { sites/giacomotognini/2020/ } \\
04 / 01 / \text { coronavirus- } \\
\text { business-tracker-how-the- } \\
\text { private-sector-is-fighting- } \\
\text { the-covid-19-pandemic/ } \\
\text { ?sh=60e6572a5899 }\end{array}$ \\
\hline
\end{tabular}

Digital Government: Research and Practice, Vol. 2, No. 2, Article 17. Publication date: December 2020. 


\begin{tabular}{|c|c|c|c|c|c|c|c|c|c|c|}
\hline 146 & Barbour & $\begin{array}{l}\text { Retail } \\
\text { Company }\end{array}$ & $\begin{array}{l}\text { Hospital } \\
\text { Gowns }\end{array}$ & Link & 01-04-2020 & England & 01-04-2020 & 1894 & Yes & $\begin{array}{l}\text { https://www.forbes.com/ } \\
\text { sites/giacomotognini/2020/ } \\
\text { 04/01/coronavirus- } \\
\text { business-tracker-how-the- } \\
\text { private-sector-is-fighting- } \\
\text { the-covid-19-pandemic/ } \\
\text { ?sh=60e6572a5899 }\end{array}$ \\
\hline 147 & $\begin{array}{l}\text { Bauer } \\
\text { Hockey }\end{array}$ & Sporting Goods & Face Shields & Link & 01-04-2020 & England & 01-04-2020 & 1927 & Yes & $\begin{array}{l}\text { https://www.forbes.com/ } \\
\text { sites/giacomotognini/2020/ } \\
\text { 04/01/coronavirus- } \\
\text { business-tracker-how-the- } \\
\text { private-sector-is-fighting- } \\
\text { the-covid-19-pandemic/ } \\
\text { ?sh=60e6572a5899 }\end{array}$ \\
\hline 148 & Brew Dog & Brewing & $\begin{array}{l}\text { Hand } \\
\text { Sanitizer }\end{array}$ & Link & 01-04-2020 & Scotland & 01-04-2020 & 2007 & No Data & $\begin{array}{l}\text { https://www.forbes.com/ } \\
\text { sites/giacomotognini/2020/ } \\
\text { 04/01/coronavirus- } \\
\text { business-tracker-how-the- } \\
\text { private-sector-is-fighting- } \\
\text { the-covid-19-pandemic/ } \\
\text { ?sh=60e6572a5899 }\end{array}$ \\
\hline 149 & Airbus & Aerospace & Ventilators & Link & $30-03-2020$ & Netherlands & $30-03-2020$ & 1970 & No Data & $\begin{array}{l}\text { https://www.smiths.com/ } \\
\text { news-and-media/2020/03/ } \\
\text { ventilatorchallengeuk- } \\
\text { consortium }\end{array}$ \\
\hline 150 & BAE Systems & $\begin{array}{l}\text { Aerospace, } \\
\text { Arms and } \\
\text { Information } \\
\text { security }\end{array}$ & Ventilators & Link & $30-03-2020$ & England & $30-03-2020$ & 1999 & Yes & $\begin{array}{l}\text { https://www.smiths.com/ } \\
\text { news-and-media/2020/03/ } \\
\text { ventilatorchallengeuk- } \\
\text { consortium }\end{array}$ \\
\hline 151 & $\begin{array}{l}\text { Bloom } \\
\text { Energy }\end{array}$ & $\begin{array}{l}\text { Fuel Cell } \\
\text { manufacturers }\end{array}$ & Ventilators & Link & $28-03-2020$ & USA & $28-03-2020$ & 2001 & Yes & $\begin{array}{l}\text { https://www.bloomenergy. } \\
\text { com/newsroom/press- } \\
\text { releases/bloom-energy- } \\
\text { state-california-almo- } \\
\text { partner-refurbish- } \\
\text { ventilators-healthcare }\end{array}$ \\
\hline 152 & BreathDirect & $\begin{array}{l}\text { Medical } \\
\text { equipment }\end{array}$ & Ventilators & Link & $27-03-2020$ & USA & $27-03-2020$ & 1997 & Yes & $\begin{array}{l}\text { https://www.forbes.com/ } \\
\text { sites/amyfeldman/2020/03/ } \\
\text { 27/medical-device- } \\
\text { entrepreneur-designs- } \\
\text { emergency-ventilator-at- } \\
\text { 10000-price-point-to- } \\
\text { battle-coronavirus/?sh= } \\
\text { 3176f4b12611 }\end{array}$ \\
\hline 153 & Dyson & $\begin{array}{l}\text { Consumer } \\
\text { Appliances }\end{array}$ & Ventilators & Link & 26-03-2020 & England & $\begin{array}{c}\text { Not } \\
\text { mentioned }\end{array}$ & 1991 & No Data & $\begin{array}{l}\text { https://www.forbes. } \\
\text { com/sites/daviddawkins/ } \\
\text { 2020/03/26/billionaire- } \\
\text { james-dyson-confirms- } \\
\text { initial-order-of-10000- } \\
\text { ventilators-will-made-in- } \\
\text { britain-at-raf-hullavington/ } \\
\text { ?utm_source= } \\
\text { followingimmediate\& } \\
\text { utm_medium=email\& } \\
\text { utm_campaign=20200326\& } \\
\text { sh=d07803616608 }\end{array}$ \\
\hline 154 & $\begin{array}{l}\text { GKN } \\
\text { Aerospace }\end{array}$ & Aerospace & Ventilators & Link & $30-03-2020$ & England & $30-03-2020$ & 1902 & Yes & $\begin{array}{l}\text { https://www.smiths.com/ } \\
\text { news-and-media/2020/03/ } \\
\text { ventilatorchallengeuk- } \\
\text { consortium }\end{array}$ \\
\hline 155 & $\begin{array}{l}\text { Inspiration } \\
\text { Healthcare }\end{array}$ & $\begin{array}{l}\text { Medical } \\
\text { Technology }\end{array}$ & Ventilators & Link & $30-03-2020$ & England & $30-03-2020$ & 2003 & Yes & $\begin{array}{l}\text { https://www.smiths.com/ } \\
\text { news-and-media/2020/03/ } \\
\text { ventilatorchallengeuk- } \\
\text { consortium }\end{array}$ \\
\hline
\end{tabular}

Digital Government: Research and Practice, Vol. 2, No. 2, Article 17. Publication date: December 2020. 


\begin{tabular}{|c|c|c|c|c|c|c|c|c|c|c|}
\hline 156 & Medtronic & $\begin{array}{l}\text { Medical } \\
\text { equipment }\end{array}$ & Ventilators & Link & $30-03-2020$ & Ireland & $30-03-2020$ & 1949 & Yes & $\begin{array}{l}\text { https://www.smiths.com/ } \\
\text { news-and-media/2020/03/ } \\
\text { ventilatorchallengeuk- } \\
\text { consortium }\end{array}$ \\
\hline 157 & Meggitt & $\begin{array}{l}\text { Aerospace } \\
\text { defence }\end{array}$ & Ventilators & Link & $30-03-2020$ & England & $30-03-2020$ & 1947 & Yes & $\begin{array}{l}\text { https://www.smiths.com/ } \\
\text { news-and-media/2020/03/ } \\
\text { ventilatorchallengeuk- } \\
\text { consortium }\end{array}$ \\
\hline 158 & Penlon & $\begin{array}{l}\text { Medical } \\
\text { equipment }\end{array}$ & Ventilators & Link & $30-03-2020$ & England & $30-03-2020$ & 1943 & Yes & $\begin{array}{l}\text { https://www.smiths.com/ } \\
\text { news-and-media/2020/03/ } \\
\text { ventilatorchallengeuk- } \\
\text { consortium }\end{array}$ \\
\hline 159 & Philips & $\begin{array}{l}\text { Consumer } \\
\text { Appliances }\end{array}$ & Ventilators & Link & $22-03-2020$ & Netherlands & $22-03-2020$ & 1891 & No Data & $\begin{array}{l}\text { https://www.nasdaq.com/ } \\
\text { articles/philips-to-ramp- } \\
\text { up-production-of- } \\
\text { ventilators-in-coronavirus- } \\
\text { fight-2020-03-22 }\end{array}$ \\
\hline 160 & ResMed & Medical & Ventilators & Link & 23-03-2020 & USA & 23-03-2020 & 1989 & Yes & $\begin{array}{l}\text { https://newsroom.resmed. } \\
\text { com/news-releases/news- } \\
\text { details/2020/ResMed- } \\
\text { Statement-on-COVID-19/ } \\
\text { default.aspx }\end{array}$ \\
\hline 161 & Rolls-Royce & Automotive & Ventilators & Link & $30-03-2020$ & England & $30-03-2020$ & 2011 & Yes & $\begin{array}{l}\text { https://www.smiths.com/ } \\
\text { news-and-media/2020/03/ } \\
\text { ventilatorchallengeuk- } \\
\text { consortium }\end{array}$ \\
\hline 162 & $\begin{array}{l}\text { Smiths } \\
\text { Group }\end{array}$ & Engineering & Ventilators & Link & $30-03-2020$ & England & $30-03-2020$ & 1851 & Yes & $\begin{array}{l}\text { https://www.smiths.com/ } \\
\text { news-and-media/2020/03/ } \\
\text { ventilatorchallengeuk- } \\
\text { consortium }\end{array}$ \\
\hline 163 & SpaceX & Aerospace & $\begin{array}{l}\text { Ventilator } \\
\text { component }\end{array}$ & Link & 01-04-2020 & USA & 01-04-2020 & 2002 & Yes & $\begin{array}{l}\text { https://www.forbes.com/ } \\
\text { sites/giacomotognini/2020/ } \\
\text { 04/01/coronavirus- } \\
\text { business-tracker-how-the- } \\
\text { private-sector-is-fighting- } \\
\text { the-covid-19-pandemic/ } \\
\text { ?sh=1479e7c65899 }\end{array}$ \\
\hline 164 & Thales Group & $\begin{array}{l}\text { Aerospace, } \\
\text { Defence, } \\
\text { Transport, } \\
\text { Security }\end{array}$ & Ventilators & Link & $30-03-2020$ & France & $30-03-2020$ & 2000 & Yes & $\begin{array}{l}\text { https://www.smiths.com/ } \\
\text { news-and-media/2020/03/ } \\
\text { ventilatorchallengeuk- } \\
\text { consortium }\end{array}$ \\
\hline 165 & $\begin{array}{l}\text { Ultra } \\
\text { Electronics }\end{array}$ & $\begin{array}{l}\text { Defence, } \\
\text { security, } \\
\text { critical } \\
\text { detection \& } \\
\text { control } \\
\end{array}$ & Ventilators & Link & $30-03-2020$ & England & $30-03-2020$ & 1920 & Yes & $\begin{array}{l}\text { https://www.smiths.com/ } \\
\text { news-and-media/2020/03/ } \\
\text { ventilatorchallengeuk- } \\
\text { consortium }\end{array}$ \\
\hline 166 & $\begin{array}{l}\text { Virgin } \\
\text { Galactic }\end{array}$ & $\begin{array}{l}\text { Spaceflight } \\
\text { Company }\end{array}$ & Ventilators & Link & 29-04-2020 & England & 29-04-2020 & 2004 & Yes & $\begin{array}{l}\text { https://www.forbes.com/ } \\
\text { sites/valeriestimac/2020/04/ } \\
\text { 29/virgin-galactic-nasa- } \\
\text { join-forces-to-fight-covid- } \\
\text { 19/?sh=1279a1937f31 }\end{array}$ \\
\hline 167 & Xerox & $\begin{array}{l}\text { Buisness and } \\
\text { Technology } \\
\text { Solution }\end{array}$ & Ventilators & Link & 06-04-2020 & USA & 06-04-2020 & 1906 & Yes & $\begin{array}{l}\text { https://www. } \\
\text { forbes.com/sites/ } \\
\text { amyfeldman/2020/04/06/ } \\
\text { xerox-will-mass-produce- } \\
\text { disposable-ventilators-in- } \\
\text { partnership-with-vortran- } \\
\text { to-fight-coronavirus/?sh= } \\
\text { 2219cca8205a }\end{array}$ \\
\hline
\end{tabular}

Digital Government: Research and Practice, Vol. 2, No. 2, Article 17. Publication date: December 2020. 


\begin{tabular}{|c|c|c|c|c|c|c|c|c|c|c|}
\hline 168 & Koniku & Neurotechnolog & $\begin{array}{l}\text { Odorant } \\
\text { Receptors }\end{array}$ & Link & 04-05-2020 & Nigeria & 04-05-2020 & 2015 & No & $\begin{array}{l}\text { https://www.airbus.com/ } \\
\text { newsroom/press-releases/ } \\
\text { en/2020/05/airbus-and- } \\
\text { koniku-inc-embark-on- } \\
\text { disruptive-biotechnology- } \\
\text { solutions-for-aviation- } \\
\text { security-operations.html }\end{array}$ \\
\hline 169 & Globe Keeper & $\begin{array}{l}\text { Tactical and } \\
\text { strategic } \\
\text { operations } \\
\text { platform }\end{array}$ & $\begin{array}{l}\text { Monitoring } \\
\text { App }\end{array}$ & Link & 01-04-2020 & Israel & 01-04-2020 & 2014 & No & $\begin{array}{l}\text { https://www.reuters. } \\
\text { com/article/us-health- } \\
\text { coronavirus-israel-apps/1- } \\
\text { 5-million-israelis-using- } \\
\text { voluntary-coronavirus- } \\
\text { monitoring-app- } \\
\text { idUSKBN21J5L5 }\end{array}$ \\
\hline 170 & \begin{tabular}{|l} 
Boston \\
Dynamics
\end{tabular} & $\begin{array}{l}\text { Engineering } \\
\text { and Robotics }\end{array}$ & Robotic Dogs & Link & 01-04-2020 & USA & 01-04-2020 & 1992 & Yes & $\begin{array}{l}\text { https://www.reuters. } \\
\text { com/article/us-health- } \\
\text { coronavirus-israel-apps/1- } \\
\text { 5-million-israelis-using- } \\
\text { voluntary-coronavirus- } \\
\text { monitoring-app- } \\
\text { idUSKBN21J5L5 }\end{array}$ \\
\hline 171 & NeuroFlow & $\begin{array}{l}\text { Software } \\
\text { Company }\end{array}$ & $\begin{array}{l}\text { Onine Tool } \\
\text { for screening }\end{array}$ & Link & $23-03-2020$ & USA & $23-03-2020$ & 2016 & Yes & $\begin{array}{l}\text { https://www.neuroflow. } \\
\text { com/neuroflow-launches- } \\
\text { coronavirus-risk- } \\
\text { assessment/ }\end{array}$ \\
\hline 172 & Palantir & $\begin{array}{l}\text { Software } \\
\text { Company }\end{array}$ & $\begin{array}{l}\text { Tracking } \\
\text { Software }\end{array}$ & Link & $28-03-2020$ & USA & $28-03-2020$ & 2003 & Yes & $\begin{array}{l}\text { https://healthtech.blog. } \\
\text { gov.uk } / 2020 / 03 / 28 / \text { the- } \\
\text { power-of-data-in-a- } \\
\text { pandemic/ }\end{array}$ \\
\hline 173 & UiPath & $\begin{array}{l}\text { Software } \\
\text { Company }\end{array}$ & $\begin{array}{l}\text { Robotic } \\
\text { process } \\
\text { automation } \\
\text { software }\end{array}$ & Link & 07-04-2020 & USA & $07-04-2020$ & 2005 & Yes & $\begin{array}{l}\text { https://www.uipath.com/ } \\
\text { newsroom/global- } \\
\text { organizations-turning-to- } \\
\text { rpa-to-respond-to-covid- } \\
\text { 19-pandemic }\end{array}$ \\
\hline 174 & UnitedHealth & $\begin{array}{l}\text { Biotechnology } \\
\text { Company }\end{array}$ & $\begin{array}{l}\text { Screening } \\
\text { app }\end{array}$ & Link & $15-03-2020$ & USA & $15-03-2020$ & 1977 & Yes & $\begin{array}{l}\text { https://news.microsoft. } \\
\text { com/2020/05/15/ } \\
\text { unitedhealth-group-and- } \\
\text { microsoft-collaborate-to- } \\
\text { launch-protectwell- } \\
\text { protocol-and-app-to- } \\
\text { support-return-to- } \\
\text { workplace-planning-and- } \\
\text { covid-19-symptom- } \\
\text { screening/ } \\
\end{array}$ \\
\hline 175 & Ava & $\begin{array}{l}\text { Biotechnology } \\
\text { Company }\end{array}$ & $\begin{array}{l}\text { Bracelete for } \\
\text { Tracking }\end{array}$ & Link & $15-04-2020$ & USA & $15-04-2020$ & 2007 & Yes & $\begin{array}{l}\text { https: } \\
\text { //www.avawomen.com/ } \\
\text { press/liechtenstein-study- } \\
\text { aims-to-help-combat- } \\
\text { coronavirus-pandemic/ }\end{array}$ \\
\hline 176 & $\begin{array}{l}\text { Credo } \\
\text { Diagnostics }\end{array}$ & $\begin{array}{l}\text { Biotechnology } \\
\text { Company }\end{array}$ & Test Kits & Link & $17-03-2020$ & Singapore & $\begin{array}{c}\text { Not } \\
\text { mentioned }\end{array}$ & 2011 & No Data & $\begin{array}{l}\text { https://www. } \\
\text { credodxbiomed.com/en/ } \\
\text { news/100-credo- } \\
\text { diagnostics-biomedical- } \\
\text { vitapcr-sars-cov-2-assay- } \\
\text { garners-ce-mark }\end{array}$ \\
\hline 177 & DiaSorin & $\begin{array}{l}\text { Biotechnology } \\
\text { Company }\end{array}$ & Test Kits & Link & $20-03-2020$ & USA & $\begin{array}{c}\text { Not } \\
\text { mentioned }\end{array}$ & 2000 & No Data & $\begin{array}{l}\text { https://www.diasorin.com/ } \\
\text { en/news/diasorin- covid-19- } \\
\text { test-has-received-fda- } \\
\text { emergency-use- } \\
\text { authorization }\end{array}$ \\
\hline 178 & $\begin{array}{l}\text { Ipsum } \\
\text { Diagnostics }\end{array}$ & $\begin{array}{l}\text { Biotechnology } \\
\text { Company }\end{array}$ & Test Kits & Link & 01-04-2020 & USA & 01-04-2020 & 2018 & Yes & $\begin{array}{l}\text { https://www.fda.gov/ } \\
\text { media/136618/download }\end{array}$ \\
\hline
\end{tabular}

Digital Government: Research and Practice, Vol. 2, No. 2, Article 17. Publication date: December 2020. 


\begin{tabular}{|c|c|c|c|c|c|c|c|c|c|c|}
\hline 179 & $\begin{array}{l}\text { Mammoth } \\
\text { Biosciences }\end{array}$ & $\begin{array}{l}\text { Biotechnology } \\
\text { Company }\end{array}$ & $\begin{array}{l}\text { Strip Tool for } \\
\text { Detection }\end{array}$ & Link & $15-02-2020$ & USA & $15-02-2020$ & 2017 & Yes & $\begin{array}{l}\text { https: } \\
\text { //mammoth.bio/2020/02/15/ } \\
\text { white-paper-a-protocol- } \\
\text { for-rapid-detection-of- } \\
\text { sars-cov-2-using-crispr- } \\
\text { sars-cov-2-detectr/ }\end{array}$ \\
\hline 180 & \begin{tabular}{|l} 
Siemens \\
Healthineers
\end{tabular} & $\begin{array}{l}\text { Biotechnology } \\
\text { Company }\end{array}$ & $\begin{array}{l}\text { Blood Gas } \\
\text { Analyser }\end{array}$ & Link & $31-03-2020$ & Germany & $31-03-2020$ & 2015 & Yes & $\begin{array}{l}\text { https://www.siemens- } \\
\text { healthineers.com/press- } \\
\text { room/press-releases/rapid } \\
\text { point500ebloodgasanalyzer. } \\
\text { html }\end{array}$ \\
\hline 181 & Fanatics & \begin{tabular}{|l|} 
Consumer \\
Appliances
\end{tabular} & $\begin{array}{l}\text { Face Masks, } \\
\text { Hospital } \\
\text { Gowns }\end{array}$ & Link & $27-03-2020$ & USA & $27-03-2020$ & 1995 & Yes & \begin{tabular}{|l} 
https://twitter.com/ \\
MichaelGRubin/status/ \\
1243258525066891264?s=20
\end{tabular} \\
\hline 182 & ExxonMobil & $\begin{array}{l}\text { Consumer } \\
\text { Appliances }\end{array}$ & $\begin{array}{l}\text { Face Shields, } \\
\text { Face Masks }\end{array}$ & Link & 02-04-2020 & USA & $\begin{array}{c}\text { Not } \\
\text { mentioned }\end{array}$ & 1999 & No Data & \begin{tabular}{|l} 
https://corporate. \\
exxonmobil.com/News/ \\
Newsroom/News-releases/ \\
2020/0402_ExxonMobil- \\
joins-Global-Center-to- \\
expedite-medical- \\
innovation-for-PPE
\end{tabular} \\
\hline 183 & Dickies & $\begin{array}{l}\text { Consumer } \\
\text { Appliances }\end{array}$ & $\begin{array}{l}\text { Hospital } \\
\text { Gowns }\end{array}$ & Link & $16-04-2020$ & USA & $16-04-2020$ & 1922 & Yes & $\begin{array}{l}\text { https://www.vfc.com/news/ } \\
\text { press-release/1719/dickies- } \\
\text { vf-corporation-to-produce- } \\
\text { 3-4-million-isolation }\end{array}$ \\
\hline 184 & GVS & $\begin{array}{l}\text { Consumer } \\
\text { Appliances }\end{array}$ & $\begin{array}{l}\text { Face Masks, } \\
\text { Ventilator } \\
\text { component }\end{array}$ & Link & $16-03-2020$ & Italy & $16-03-2020$ & 1984 & Yes & $\begin{array}{l}\text { https://www.forbes. } \\
\text { com/sites/giacomotognini/ } \\
\text { 2020/03/16/this-italian- } \\
\text { companys-masks-and- } \\
\text { filters-are-helping-doctors- } \\
\text { fight-back-against-the- } \\
\text { coronavirusin-china-the- } \\
\text { us-italy-and-beyond/?sh= } \\
\text { 97ddb4a7b6c3 }\end{array}$ \\
\hline 185 & $\begin{array}{l}\text { Charoen } \\
\text { Popkhand } \\
\text { Group }\end{array}$ & $\begin{array}{l}\text { Consumer } \\
\text { Appliances }\end{array}$ & $\begin{array}{l}\text { Surgical } \\
\text { Masks }\end{array}$ & Link & 01-04-2020 & Thailand & $\begin{array}{c}\text { Not } \\
\text { mentioned }\end{array}$ & 1921 & No Data & $\begin{array}{l}\text { htttps://www.forbes.com/ } \\
\text { sites/gracechung/2020/04/ } \\
\text { 13/forbes-asia-tracker- } \\
\text { how-the-regions-tycoons- } \\
\text { entrepreneurs-and- } \\
\text { businesses-are-combating- } \\
\text { the-coronavirus/?sh= } \\
\text { 62be646b2b64 }\end{array}$ \\
\hline 186 & $\begin{array}{l}\text { Cantabria } \\
\text { Lab }\end{array}$ & \begin{tabular}{|l} 
Consumer \\
Appliances
\end{tabular} & $\begin{array}{l}\text { Hand } \\
\text { Sanitizer }\end{array}$ & Link & 19-03-2020 & Spain & $\begin{array}{c}\text { Not } \\
\text { mentioned }\end{array}$ & 1989 & No Data & $\begin{array}{l}\text { https://www.cantabrialabs. } \\
\text { com/en/communication/ } \\
\text { cantabria-labs-starts- } \\
\text { making-hand-sanitiser-for- } \\
\text { health-personnel/ }\end{array}$ \\
\hline 187 & $\begin{array}{l}\text { Canada } \\
\text { Goose }\end{array}$ & $\begin{array}{l}\text { Consumer } \\
\text { Appliances }\end{array}$ & $\begin{array}{l}\text { Medical } \\
\text { Scrubs, } \\
\text { Hospital } \\
\text { Gowns }\end{array}$ & Link & $25-03-2020$ & Canada & 25-03-2020 & 1957 & No Data & $\begin{array}{l}\text { https://www.canadagoose. } \\
\text { com/on/demandware.static/ } \\
\text {-/Library-Sites-CG-Global/ } \\
\text { default/dw40fd0561/press/ } \\
\text { Canada-Goose-Dedicates- } \\
\text { Manufacturing-Resources- } \\
\text { in-Fight-Against-COVID- } \\
\text { 19.pdf }\end{array}$ \\
\hline 188 & $\begin{array}{l}\text { Calzedonia } \\
\text { Group }\end{array}$ & $\begin{array}{l}\text { Consumer } \\
\text { Appliances }\end{array}$ & $\begin{array}{l}\text { Face Masks, } \\
\text { Hospital } \\
\text { Gowns }\end{array}$ & Link & 29-05-2020 & Italy & 29-05-2020 & 1986 & No & $\begin{array}{l}\text { https://www. } \\
\text { calzedoniagroup.com/en/ } \\
\text { news-archive/news-6 }\end{array}$ \\
\hline 189 & Bvlgari & $\begin{array}{l}\text { Consumer } \\
\text { Appliances }\end{array}$ & $\begin{array}{l}\text { Hand } \\
\text { Sanitizer }\end{array}$ & Link & 02-04-2020 & Italy & $\begin{array}{c}\text { Not } \\
\text { mentioned }\end{array}$ & 1884 & No Data & $\begin{array}{l}\text { https://www.bulgari.com/ } \\
\text { en-us/stories/bulgari- } \\
\text { donate-hand-sanitizer- } \\
\text { against-coronavirus.html }\end{array}$ \\
\hline
\end{tabular}

Digital Government: Research and Practice, Vol. 2, No. 2, Article 17. Publication date: December 2020. 


\begin{tabular}{|c|c|c|c|c|c|c|c|c|c|c|}
\hline 190 & Fippi & $\begin{array}{l}\text { Consumer } \\
\text { Appliances }\end{array}$ & Face Masks & Link & $29-03-2020$ & Italy & $29-03-2020$ & 1970 & Yes & $\begin{array}{l}\text { https://twitter.com/ } \\
\text { RegLombardia/status/ } \\
1244284212976459778\end{array}$ \\
\hline 191 & GelPro & $\begin{array}{l}\text { Consumer } \\
\text { Appliances }\end{array}$ & Face Shields & Link & 01-04-2020 & USA & 01-04-2020 & 2002 & Yes & $\begin{array}{l}\text { https://www.statesman. } \\
\text { com/business/20200401/ } \\
\text { austinrsquos-gelpro- } \\
\text { making-face-shields-to- } \\
\text { aid-in-coronavirus-fight }\end{array}$ \\
\hline 192 & HP & $\begin{array}{l}\text { Consumer } \\
\text { Appliances }\end{array}$ & $\begin{array}{l}\text { Face Masks, } \\
\text { Face Shields, } \\
\text { Ventilator } \\
\text { component }\end{array}$ & Link & $24-03-2020$ & Spain & $24-03-2020$ & 1939 & Yes & $\begin{array}{l}\text { https://press.hp.com/us/en/ } \\
\text { press-releases/2020/hp-inc- } \\
\text { and-partners-mobilize-3d- } \\
\text { printing-solutions-to- } \\
\text { battle-c.html }\end{array}$ \\
\hline 193 & INEOS & $\begin{array}{l}\text { Consumer } \\
\text { Appliances }\end{array}$ & $\begin{array}{l}\text { Hand } \\
\text { Sanitizer }\end{array}$ & Link & 24-03-2020 & Scotland & $24-03-2020$ & 1998 & No Data & $\begin{array}{l}\text { https://press.hp.com/us/en/ } \\
\text { press-releases/2020/hp-inc- } \\
\text { and-partners-mobilize-3d- } \\
\text { printing-solutions-to- } \\
\text { battle-c.html }\end{array}$ \\
\hline 194 & $\begin{array}{l}\text { Jenco } \\
\text { Productions }\end{array}$ & $\begin{array}{l}\text { Consumer } \\
\text { Appliances }\end{array}$ & Face Shields & Link & 01-04-2020 & USA & 01-04-2020 & 1995 & Yes & $\begin{array}{l}\text { https://www. } \\
\text { jencoproductions.com/ }\end{array}$ \\
\hline 195 & Jockey & $\begin{array}{l}\text { Retail } \\
\text { Company }\end{array}$ & $\begin{array}{l}\text { Face Masks, } \\
\text { Hospital } \\
\text { Gowns }\end{array}$ & Link & 01-04-2020 & USA & 01-04-2020 & 1876 & Yes & $\begin{array}{l}\text { https://www.forbes. } \\
\text { com/sites/giacomotognini/ } \\
\text { 2020/04/01/coronavirus- } \\
\text { business-tracker-how-the- } \\
\text { private-sector-is-fighting- } \\
\text { the-covid-19-pandemic/ } \\
\text { ?sh=3f6067ff5899 }\end{array}$ \\
\hline 196 & Decathlon & $\begin{array}{l}\text { Retail } \\
\text { Company }\end{array}$ & Respirators & Link & $14-04-2020$ & France & $\begin{array}{c}\text { Not } \\
\text { mentioned }\end{array}$ & 1976 & No Data & $\begin{array}{l}\text { https://www.decathlon. } \\
\text { com/blogs/inside- } \\
\text { decathlon/decathlon- } \\
\text { easybreath-mask-covid-19- } \\
\text { update }\end{array}$ \\
\hline 197 & $\begin{array}{l}\text { Orient } \\
\text { Electric }\end{array}$ & $\begin{array}{l}\text { Consumer } \\
\text { Appliances }\end{array}$ & $\begin{array}{l}\text { UVC } \\
\text { Disinfection } \\
\text { Product }\end{array}$ & Link & $18-07-2020$ & India & $18-07-2020$ & 1954 & No & $\begin{array}{l}\text { https: } \\
\text { //www.=moneycontrol. } \\
\text { com/news/business/orient- } \\
\text { electric-enters-health- } \\
\text { appliances-space-launches- } \\
\text { uv-sanitiser-device- } \\
\text { 5559621.html }\end{array}$ \\
\hline 198 & Borosil & $\begin{array}{l}\text { Consumer } \\
\text { Appliances }\end{array}$ & $\begin{array}{l}\text { UVC } \\
\text { Disinfection } \\
\text { Product }\end{array}$ & Link & $22-07-2020$ & India & $22-07-2020$ & 1962 & No & $\begin{array}{l}\text { https://gadgets.ndtv.com/ } \\
\text { others/news/borosil- } \\
\text { suraksha-uv-disinfection- } \\
\text { price-in-india-rs-11990- } \\
\text { launch-specifications- } \\
\text { features-2267039 }\end{array}$ \\
\hline 199 & Finolex & $\begin{array}{l}\text { Consumer } \\
\text { Appliances }\end{array}$ & $\begin{array}{l}\text { Hand } \\
\text { Sanitizer }\end{array}$ & Storefront & $23-07-2020$ & India & $\begin{array}{c}\text { Not } \\
\text { mentioned }\end{array}$ & 1958 & No Data & Storefront \\
\hline 200 & Vystar & $\begin{array}{l}\text { Consumer } \\
\text { Appliances }\end{array}$ & $\begin{array}{l}\text { UVC } \\
\text { Disinfection } \\
\text { Product }\end{array}$ & Link & $22-07-2020$ & USA & $22-07-2020$ & 1952 & No & $\begin{array}{l}\text { https://www. } \\
\text { globenewswire.com/news- } \\
\text { release/2020/07/22/2065910/ } \\
\text { 0/en/Vystar-Introduces- } \\
\text { RxAir400-UV-C-Light- } \\
\text { Germicidal-Air-Purifier- } \\
\text { Extended-Life-UV-Bulb- } \\
\text { Wall-Mount-Option.html }\end{array}$ \\
\hline 201 & Samsung & $\begin{array}{l}\text { Consumer } \\
\text { Appliances }\end{array}$ & $\begin{array}{l}\text { UVC } \\
\text { Disinfection } \\
\text { Product }\end{array}$ & Link & $23-07-2020$ & India & $23-07-2020$ & 1938 & No & $\begin{array}{l}\text { https://indianexpress.com/ } \\
\text { article/technology/gadgets/ } \\
\text { uv-lights-are-becoming- } \\
\text { the-weapon-of-choice-to- } \\
\text { kill-the-virus- } 6520319 /\end{array}$ \\
\hline
\end{tabular}

Digital Government: Research and Practice, Vol. 2, No. 2, Article 17. Publication date: December 2020. 


\begin{tabular}{|c|c|c|c|c|c|c|c|c|c|c|}
\hline 202 & Godrej & $\begin{array}{l}\text { Consumer } \\
\text { Appliances }\end{array}$ & $\begin{array}{l}\text { UVC } \\
\text { Disinfection } \\
\text { Product }\end{array}$ & Link & $23-07-2020$ & India & $23-07-2020$ & 1897 & No & $\begin{array}{l}\text { https://indianexpress.com/ } \\
\text { article/technology/gadgets/ } \\
\text { uv-lights-are-becoming- } \\
\text { the-weapon-of-choice-to- } \\
\text { kill-the-virus- } 6520319 /\end{array}$ \\
\hline 203 & CuraSigna & $\begin{array}{l}\text { Consumer } \\
\text { Appliances }\end{array}$ & Ventilators & Link & $23-07-2020$ & India & $23-07-2020$ & 2014 & No & $\begin{array}{l}\text { https://indianexpress.com/ } \\
\text { article/technology/gadgets/ } \\
\text { uv-lights-are-becoming- } \\
\text { the-weapon-of-choice-to- } \\
\text { kill-the-virus- } 6520319 /\end{array}$ \\
\hline 204 & Philips & $\begin{array}{l}\text { Consumer } \\
\text { Appliances }\end{array}$ & $\begin{array}{l}\text { UVC } \\
\text { Disinfection } \\
\text { Product }\end{array}$ & Link & $24-07-2020$ & India & $24-07-2020$ & 1891 & No & $\begin{array}{l}\text { https: } \\
\text { //www.news18.com/news/ } \\
\text { tech/philips-uv-c-system- } \\
\text { review-this-will-disinfect- } \\
\text { everything-you-can-fit- } \\
\text { inside-it-2731485.html }\end{array}$ \\
\hline 205 & $\begin{array}{l}\text { Muse } \\
\text { Wearables }\end{array}$ & $\begin{array}{l}\text { Consumer } \\
\text { Appliances }\end{array}$ & $\begin{array}{l}\text { Wrist based } \\
\text { tracker }\end{array}$ & Link & $25-07-2020$ & India & $25-07-2020$ & 2012 & No & $\begin{array}{l}\text { https://www.thehindu.com// } \\
\text { news/national/iit-madras- } \\
\text { startup-generates-22-cr- } \\
\text { funding-for-launching- } \\
\text { wrist-band-for-detecting- } \\
\text { covid-symptoms/ } \\
\text { article32189460.ece }\end{array}$ \\
\hline 206 & Cartoni & $\begin{array}{l}\text { Consumer } \\
\text { Appliances }\end{array}$ & $\begin{array}{l}\text { UVC } \\
\text { Disinfection } \\
\text { Product }\end{array}$ & Link & $27-07-2020$ & Italy & $27-07-2020$ & 1935 & No & $\begin{array}{l}\text { https://www.newsshooter. } \\
\text { com/2020/07/27/cartoni- } \\
\text { uv-c-boxer-uv- } \\
\text { disinfection-device-for- } \\
\text { video-motion-picture- } \\
\text { equipment/ }\end{array}$ \\
\hline 207 & $\begin{array}{l}\text { Bharat } \\
\text { Electronics } \\
\text { Ltd }\end{array}$ & $\begin{array}{l}\text { Consumer } \\
\text { Appliances }\end{array}$ & Ventilators & Link & $30-03-2020$ & India & $30-03-2020$ & 1954 & Yes & $\begin{array}{l}\text { https://www.thehindu.com// } \\
\text { news/national/automobile- } \\
\text { makers-asked-to-built- } \\
\text { ventilators-health- } \\
\text { ministry/article31204808. } \\
\text { ece }\end{array}$ \\
\hline 208 & $\begin{array}{l}\text { The Trident } \\
\text { Group }\end{array}$ & Conglomerate & $\begin{array}{l}\text { Face Masks, } \\
\text { PPE Kits }\end{array}$ & Link & 01-05-2020 & India & 01-05-2020 & 1990 & Yes & $\begin{array}{l}\text { https://static.investindia. } \\
\text { gov.in/2020-07/ } \\
\text { PPE\%20Report_- } \\
\text { Invest\%20India.pdf }\end{array}$ \\
\hline 209 & $\begin{array}{l}\text { The Aditya } \\
\text { Birla Group }\end{array}$ & Conglomerate & $\begin{array}{l}\text { Face Masks, } \\
\text { Hospital } \\
\text { Gowns }\end{array}$ & Link & 01-05-2020 & India & 01-05-2020 & 1857 & Yes & $\begin{array}{l}\text { https://static.investindia. } \\
\text { gov.in/2020-07/ } \\
\text { PPE\%20Report_- } \\
\text { Invest\%20India.pdf }\end{array}$ \\
\hline 210 & Sure Safety & $\begin{array}{l}\text { Industrial } \\
\text { safety } \\
\text { equipment }\end{array}$ & PPE Kits & Link & 01-05-2020 & India & 01-05-2020 & 1974 & Yes & $\begin{array}{l}\text { https://static.investindia. } \\
\text { gov.in/2020-07/ } \\
\text { PPE\%20Report_- } \\
\text { Invest\%20India.pdf }\end{array}$ \\
\hline 211 & $\begin{array}{l}\text { Matrix } \\
\text { Clothing }\end{array}$ & $\begin{array}{l}\text { Garment } \\
\text { manufacturers }\end{array}$ & PPE Kits & Link & 01-05-2020 & India & 01-05-2020 & 1980 & Yes & $\begin{array}{l}\text { https://static.investindia. } \\
\text { gov.in/2020-07/ } \\
\text { PPE\%20Report_- } \\
\text { Invest\%20India.pdf }\end{array}$ \\
\hline 212 & $\begin{array}{l}\text { Shahi } \\
\text { Exports }\end{array}$ & $\begin{array}{l}\text { Garment } \\
\text { manufacturers }\end{array}$ & PPE Kits & Link & 01-05-2020 & India & 01-05-2020 & 1974 & Yes & $\begin{array}{l}\text { https://static.investindia. } \\
\text { gov.in/2020-07/ } \\
\text { PPE\%20Report_- } \\
\text { Invest\%20India.pdf }\end{array}$ \\
\hline 213 & Welspun & Conglomerate & $\begin{array}{l}\text { Hand } \\
\text { Sanitizer }\end{array}$ & Link & 01-05-2020 & India & 01-05-2020 & 1985 & Yes & $\begin{array}{l}\text { https://static.investindia. } \\
\text { gov.in/2020-07/ } \\
\text { PPE\%20Report_- } \\
\text { Invest\%20India.pdf }\end{array}$ \\
\hline
\end{tabular}

Digital Government: Research and Practice, Vol. 2, No. 2, Article 17. Publication date: December 2020. 


\begin{tabular}{|c|c|c|c|c|c|c|c|c|c|c|}
\hline 214 & $\begin{array}{l}\text { Alok } \\
\text { Industries }\end{array}$ & $\begin{array}{l}\text { Garment } \\
\text { manufacturers }\end{array}$ & PPE Kits & Link & 01-05-2020 & India & 01-05-2020 & 1986 & Yes & $\begin{array}{l}\text { https://static.investindia. } \\
\text { gov.in/2020-07/ } \\
\text { PPE\%20Report_- } \\
\text { Invest\%20India.pdf } \\
\end{array}$ \\
\hline 215 & JCT Mills & $\begin{array}{l}\text { Garment } \\
\text { manufacturers }\end{array}$ & PPE Kits & Link & $01-05-2020$ & India & 01-05-2020 & 1971 & Yes & $\begin{array}{l}\text { https://static.investindia. } \\
\text { gov.in/2020-07/ } \\
\text { PPE\%20Report_- } \\
\text { Invest\%20India.pdf }\end{array}$ \\
\hline 216 & Arvind Mills & Conglomerate & PPE Kits & Link & 01-05-2020 & India & $01-05-2020$ & 1931 & Yes & $\begin{array}{l}\text { https://static.investindia. } \\
\text { gov.in/2020-07/ } \\
\text { PPE\%20Report__ } \\
\text { Invest\%20India.pdf } \\
\end{array}$ \\
\hline 217 & \begin{tabular}{|l} 
SCG \\
Enterprises
\end{tabular} & $\begin{array}{l}\text { Chemical } \\
\text { Industry }\end{array}$ & PPE Kits & Link & 01-05-2020 & India & 01-05-2020 & 1999 & Yes & $\begin{array}{l}\text { https://static.investindia. } \\
\text { gov.in/2020-07/ } \\
\text { PPE\%20Report_- } \\
\text { Invest\%20India.pdf }\end{array}$ \\
\hline 218 & Amaryllis & $\begin{array}{l}\text { Medical } \\
\text { Healthcare }\end{array}$ & PPE Kits & Link & $01-05-2020$ & India & $01-05-2020$ & 1952 & Yes & $\begin{array}{l}\text { https://static.investindia. } \\
\text { gov.in/2020-07/ } \\
\text { PPE\%20Report_- } \\
\text { Invest\%20India.pdf }\end{array}$ \\
\hline 219 & \begin{tabular}{|l} 
Shree \\
Healthcare
\end{tabular} & $\begin{array}{l}\text { Medical } \\
\text { Healthcare }\end{array}$ & PPE Kits & Link & 01-05-2020 & India & $01-05-2020$ & 1988 & Yes & $\begin{array}{l}\text { https://static.investindia. } \\
\text { gov.in/2020-07/ } \\
\text { PPE\%20Report__ } \\
\text { Invest\%20India.pdf }\end{array}$ \\
\hline 220 & $\begin{array}{l}\text { Shiva } \\
\text { Texyarn }\end{array}$ & $\begin{array}{l}\text { Garment } \\
\text { manufacturers }\end{array}$ & PPE Kits & Link & 01-05-2020 & India & 01-05-2020 & 1980 & Yes & $\begin{array}{l}\text { https://static.investindia. } \\
\text { gov.in/2020-07/ } \\
\text { PPE\%20Report_- } \\
\text { Invest\%20India.pdf }\end{array}$ \\
\hline 221 & $\begin{array}{l}\text { Frontier } \\
\text { Protective } \\
\text { wear }\end{array}$ & $\begin{array}{l}\text { Industrial } \\
\text { safety } \\
\text { equipment }\end{array}$ & PPE Kits & Link & 01-05-2020 & India & 01-05-2020 & 1993 & Yes & $\begin{array}{l}\text { https://static.investindia. } \\
\text { gov.in/2020-07/ } \\
\text { PPE\%20Report__ } \\
\text { Invest\%20India.pdf } \\
\end{array}$ \\
\hline 222 & Sai Synergy & $\begin{array}{l}\text { Industrial } \\
\text { safety } \\
\text { equipment }\end{array}$ & PPE Kits & Link & 01-05-2020 & India & 01-05-2020 & 2009 & Yes & $\begin{array}{l}\text { https://static.investindia. } \\
\text { gov.in/2020-07/ } \\
\text { PPE\%20Report_- } \\
\text { Invest\%20India.pdf }\end{array}$ \\
\hline 223 & Swell Knit & $\begin{array}{l}\text { Garment } \\
\text { manufacturers }\end{array}$ & PPE Kits & Link & $14-04-2020$ & India & $14-04-2020$ & 2001 & Yes & $\begin{array}{l}\text { https://theprint.in/india/ } \\
\text { tirupur-moves-from-hm- } \\
\text { tommy-hilfiger-marks- } \\
\text { spencer-to-making-masks- } \\
\text { and-ppe-kits/400898/ }\end{array}$ \\
\hline 224 & \begin{tabular}{|l} 
Melange \\
Polymers
\end{tabular} & $\begin{array}{l}\text { Garment } \\
\text { manufacturers }\end{array}$ & $\begin{array}{l}\text { Face Masks, } \\
\text { PPE Kits }\end{array}$ & Link & $14-04-2020$ & India & $14-04-2020$ & 2012 & Yes & $\begin{array}{l}\text { https://theprint.in/india/ } \\
\text { tirupur-moves-from-hm- } \\
\text { tommy-hilfiger-marks- } \\
\text { spencer-to-making-masks- } \\
\text { and-ppe-kits/400898/ }\end{array}$ \\
\hline
\end{tabular}

Digital Government: Research and Practice, Vol. 2, No. 2, Article 17. Publication date: December 2020. 
17:32 - M. C. Kumari and B. Sagar

\begin{tabular}{|c|c|c|c|c|c|c|c|c|c|c|}
\hline 225 & Mobitizer & $\begin{array}{l}\text { Consumer } \\
\text { Goods }\end{array}$ & $\begin{array}{l}\text { UVC } \\
\text { Disinfection } \\
\text { Product }\end{array}$ & Link & $25-02-2020$ & India & $04-06-2020$ & 2000 & No & 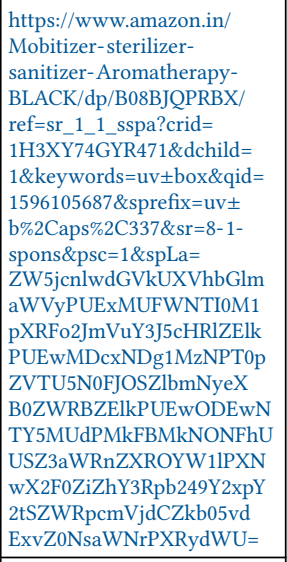 \\
\hline 226 & $\begin{array}{l}\text { Homeray-the } \\
\text { Souvenir }\end{array}$ & $\begin{array}{l}\text { Consumer } \\
\text { Goods }\end{array}$ & $\begin{array}{l}\text { UVC } \\
\text { Disinfection } \\
\text { Product }\end{array}$ & Link & $13-03-2020$ & India & $14-06-2020$ & 2012 & No &  \\
\hline 227 & Pyrotech & $\begin{array}{l}\text { Consumer } \\
\text { Appliances }\end{array}$ & $\begin{array}{l}\text { UVC } \\
\text { Disinfection } \\
\text { Product }\end{array}$ & Link & 02-04-2020 & India & $17-06-2020$ & 1976 & No & 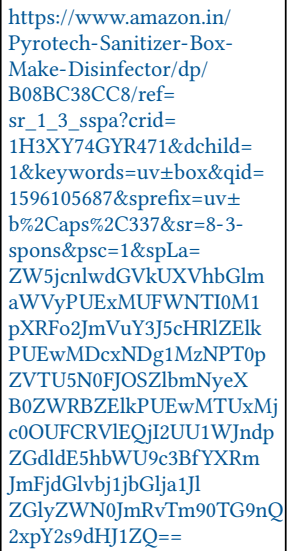 \\
\hline
\end{tabular}

Digital Government: Research and Practice, Vol. 2, No. 2, Article 17. Publication date: December 2020. 
Global Pandemic and Rapid New Product Development of Medical Products

\begin{tabular}{|c|c|c|c|c|c|c|c|c|c|c|}
\hline 228 & IFITech & $\begin{array}{l}\text { Consumer } \\
\text { Appliances }\end{array}$ & $\begin{array}{l}\text { UVC } \\
\text { Disinfection } \\
\text { Product }\end{array}$ & Link & 05-07-2020 & India & $16-05-2020$ & 2012 & Yes & $\begin{array}{l}\text { https://www.amazon.in/ } \\
\text { IFITech-Portable-sterilizer- } \\
\text { Disinfection-Scissors/dp/ } \\
\text { B089RLP1MF/ref=sr_1_5? } \\
\text { crid=1H3XY74GYR471\& } \\
\text { dchild=1\&keywords=uv } \pm \\
\text { box\&qid=1596105687\& } \\
\text { sprefixaveuv } \pm \\
\text { b\%2Caps\%2C337\&sr=8-5 }\end{array}$ \\
\hline 229 & Eco UV & $\begin{array}{l}\text { Consumer } \\
\text { Appliances }\end{array}$ & $\begin{array}{l}\text { UVC } \\
\text { Disinfection } \\
\text { Product }\end{array}$ & Link & $14-04-2020$ & USA & 02-06-2020 & 1995 & No & 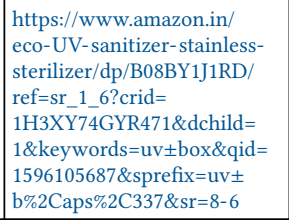 \\
\hline 230 & Smartcam & $\begin{array}{l}\text { Consumer } \\
\text { Appliances }\end{array}$ & $\begin{array}{l}\text { UVC } \\
\text { Disinfection } \\
\text { Product }\end{array}$ & Link & 04-06-2020 & India & 21-05-2020 & 2003 & Yes & $\begin{array}{l}\text { https://www.amazon.in/ } \\
\text { SmartCam-Portable- } \\
\text { Disinfection-Ultraviolet- } \\
\text { Sterilization/dp/ } \\
\text { B089N6RCH2/ref=sr_1_7? } \\
\text { crid=1H3XY74GYR471\& } \\
\text { dchild=1\&keywords=uv } \pm \\
\text { box\&qid=1596105687\& } \\
\text { sprefix=ut } \pm \\
\text { b\%2Caps\%2C337\&sr=8-7 }\end{array}$ \\
\hline 231 & Steelbird & $\begin{array}{l}\text { Consumer } \\
\text { Appliances }\end{array}$ & Face Shield & Link & $13-08-2020$ & India & $\begin{array}{c}\text { Not } \\
\text { mentioned }\end{array}$ & 1964 & No Data & $\begin{array}{l}\text { https://indianbizreview.in/ } \\
\text { 2020/08/13/steelbird- } \\
\text { launches-first-ever-hands- } \\
\text { free-face-shield-in-india/ }\end{array}$ \\
\hline 232 & BYD Co. & $\begin{array}{l}\text { Consumer } \\
\text { Appliances }\end{array}$ & Face Masks & Link & $13-03-2020$ & USA & $\begin{array}{c}\text { Not } \\
\text { mentioned }\end{array}$ & 1981 & No Data & $\begin{array}{l}\text { https://www.bloomberg. } \\
\text { com/news/articles/2020-03- } \\
13 / \text { buffett-backed-byd- } \\
\text { says-it-s-now-the-biggest- } \\
\text { maker-of-face-masks }\end{array}$ \\
\hline 233 & Godrej & $\begin{array}{l}\text { Consumer } \\
\text { Appliances }\end{array}$ & $\begin{array}{l}\text { UVC } \\
\text { Disinfection } \\
\text { Product }\end{array}$ & Link & $10-08-2020$ & India & $\begin{array}{c}\text { Not } \\
\text { mentioned }\end{array}$ & 1897 & No Data & \begin{tabular}{|l|} 
https://www. \\
financialexpress.com/ \\
industry/technology/ \\
godrej-uv-case-handy- \\
device-for-sanitising-and- \\
keeping-pathogens-at-bay/ \\
2049845/\#: :text= \\
The\%20UV\%20Case\%20by\%20 \\
Godrej, \\
bags\%20and\%20many \\
\%20other\%20articles
\end{tabular} \\
\hline 234 & $\begin{array}{l}\text { Orient } \\
\text { Electric }\end{array}$ & $\begin{array}{l}\text { Consumer } \\
\text { Appliances }\end{array}$ & $\begin{array}{l}\text { UVC } \\
\text { Disinfection } \\
\text { Product }\end{array}$ & Link & 7/17/2020 & India & $\begin{array}{c}\text { Not } \\
\text { mentioned }\end{array}$ & 1954 & No Data & $\begin{array}{l}\text { https://economictimes. } \\
\text { indiatimes.com/industry/ } \\
\text { cons-products/fmcg/orient- } \\
\text { electric-enters-hygiene- } \\
\text { products-segment/ } \\
\text { articleshow/77013802.cms? } \\
\text { from=mdr }\end{array}$ \\
\hline 235 & Lifebuoy & $\begin{array}{l}\text { Consumer } \\
\text { Goods }\end{array}$ & $\begin{array}{l}\text { Hand } \\
\text { Sanitizer }\end{array}$ & Link & $14-09-2020$ & India & $\begin{array}{c}\text { Not } \\
\text { mentioned }\end{array}$ & 1895 & No Data & $\begin{array}{l}\text { https://www.lifebuoy.in/ } \\
\text { products/sanitizer.html }\end{array}$ \\
\hline 236 & Bubble Byte & $\begin{array}{l}\text { Engineering } \\
\text { and } \\
\text { Technology }\end{array}$ & $\begin{array}{l}\text { Hand } \\
\text { Sanitizer }\end{array}$ & Link & $5 / 1 / 2020$ & India & $\begin{array}{c}\text { Not } \\
\text { mentioned }\end{array}$ & 1895 & No & $\begin{array}{l}\text { https://www.startupindia. } \\
\text { gov.in/content/dam/invest- } \\
\text { india/Templates/public/ } \\
\text { covid_resource_section/ } \\
\text { COVIDResource\& } \\
\text { Programs_ } \\
\text { Listicle\%20of\%20Covid\%20Sta } \\
\text { rtup\%20Solutions_29.04. } \\
\text { 20_86\%20shortlist.pdf }\end{array}$ \\
\hline
\end{tabular}

Digital Government: Research and Practice, Vol. 2, No. 2, Article 17. Publication date: December 2020. 


\begin{tabular}{|c|c|c|c|c|c|c|c|c|c|c|}
\hline 237 & IIT, Madras & $\begin{array}{l}\text { Engineering } \\
\text { and } \\
\text { Technology }\end{array}$ & $\begin{array}{l}\text { Hand } \\
\text { Sanitizer }\end{array}$ & Link & 01-05-2020 & India & 04-09-2020 & 2012 & No & $\begin{array}{l}\text { https://www. } \\
\text { startupindia.gov.in/ } \\
\text { content/dam/invest- } \\
\text { india/Templates/public/ } \\
\text { covid_resource_section/ } \\
\text { COVIDResource\& } \\
\text { Programs_Listicle\%20of\% } \\
\text { 20Covid\%20Startup\%20Solu } \\
\text { tions_29.04. } \\
\text { 20_86\%20shortlist.pdf }\end{array}$ \\
\hline 238 & Shapeysquare & $\begin{array}{l}\text { Consumer } \\
\text { goods }\end{array}$ & Nasal Drop & Link & 01-05-2020 & India & $10-10-2020$ & 1959 & Yes & \begin{tabular}{|l|} 
https://www. \\
startupindia.gov.in/ \\
content/dam/invest- \\
india/Templates/public/ \\
covid_resource_section/ \\
COVIDResource\& \\
Programs_ \\
Listicle\%20of\%20Covid\%20S \\
artup\%20Solutions_29.04. \\
20_86\%20shortlist.pdf \\
\end{tabular} \\
\hline 239 & \begin{tabular}{|l} 
Sunfox \\
Technology
\end{tabular} & $\begin{array}{l}\text { Research and } \\
\text { Development } \\
\text { lab }\end{array}$ & Face Shield & Link & 01-05-2020 & India & $16-04-2020$ & 2018 & Yes & \begin{tabular}{|l|} 
https://www. \\
startupindia.gov.in/ \\
content/dam/invest- \\
india/Templates/public/ \\
covid_resource_section/ \\
COVIDResource\& \\
Programs_ \\
Listicle\%20of\%20Covid\%20S \\
artup\%20Solutions_29.04. \\
20_86\%20shortlist.pdf \\
\end{tabular} \\
\hline 240 & $\begin{array}{l}\text { Swasti Agro } \\
\text { and } \\
\text { Biproducts }\end{array}$ & $\begin{array}{l}\text { Consumer } \\
\text { goods }\end{array}$ & Face Shield & Link & 01-05-2020 & India & $31-03-2020$ & 2016 & No & \begin{tabular}{|l|} 
https://www. \\
startupindia.gov.in/ \\
content/dam/invest- \\
india/Templates/public/ \\
covid_resource_section/ \\
COVIDResource\& \\
Programs_- \\
Listicle\%20of\%20Covid\%20St \\
artup\%20Solutions_29.04. \\
20_86\%20shortlist.pdf
\end{tabular} \\
\hline
\end{tabular}

Digital Government: Research and Practice, Vol. 2, No. 2, Article 17. Publication date: December 2020. 
A.2 Charts Showing the Original Product-segment Background and COVID-19 Medical Products Launched by the 240 Companies

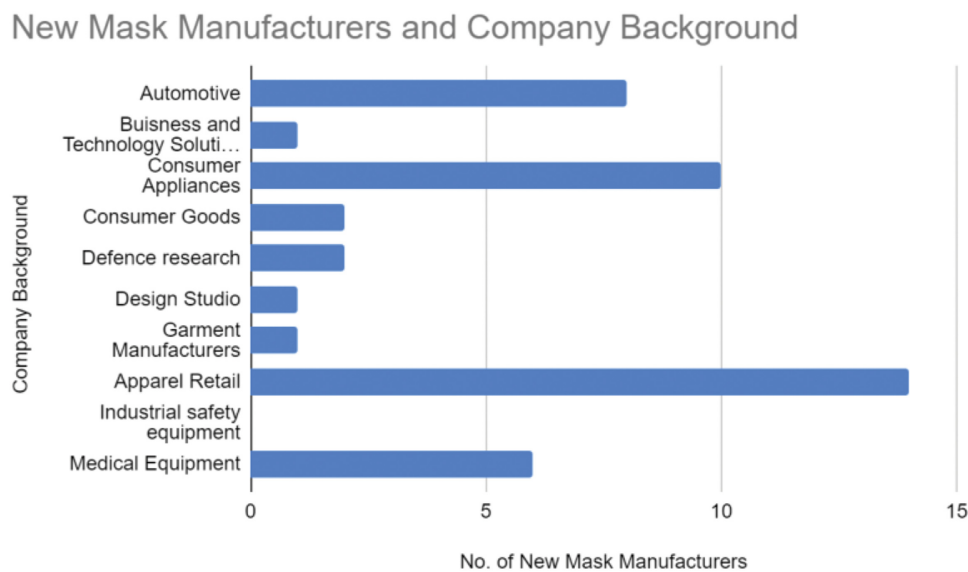

Company backgrounds of 45 new mask producers

New Sanitiser Manufacturers and Company Background

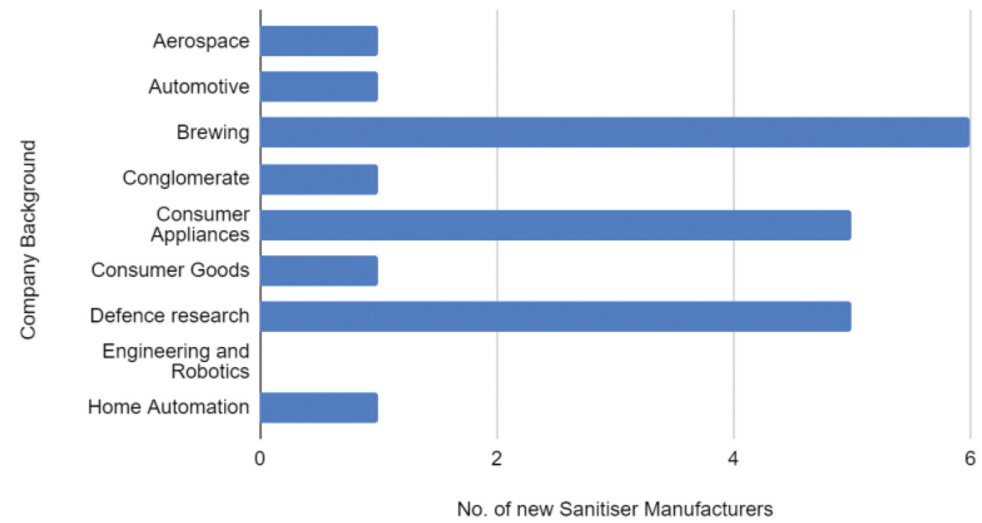

Company backgrounds of 21 new sanitizer producers 
New Face Shield Manufacturers and Company Background

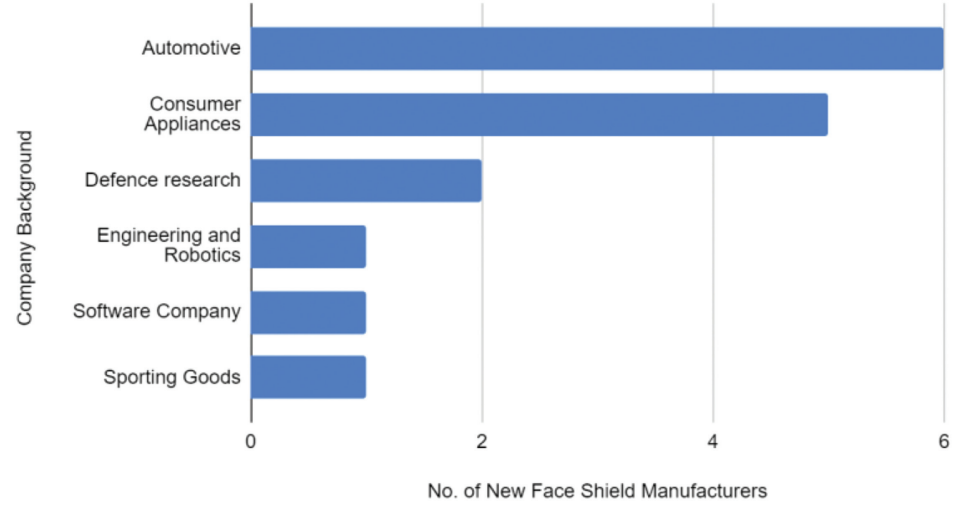

\section{Company backgrounds of 16 new face shield producers}

New Test Kits Manufacturers and Company Background

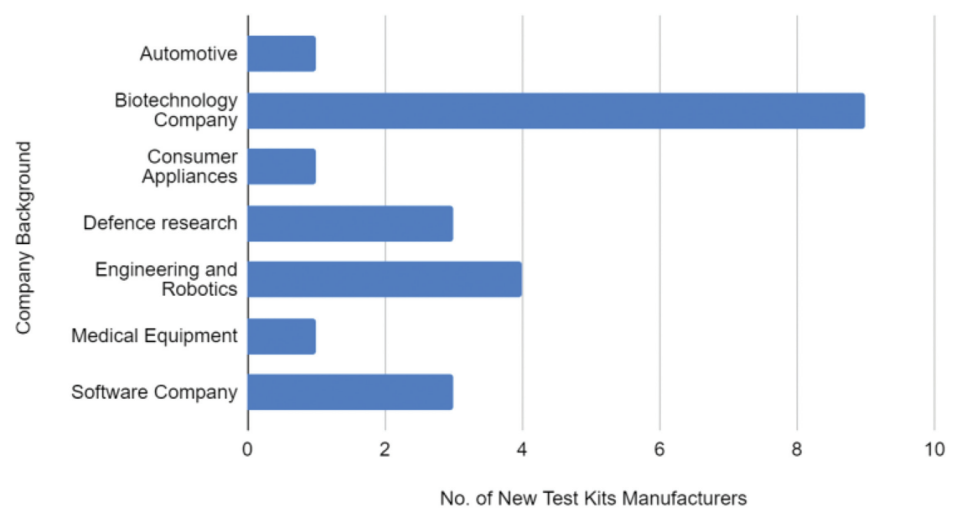

Company backgrounds of 22 new COVID-19 test kit producers

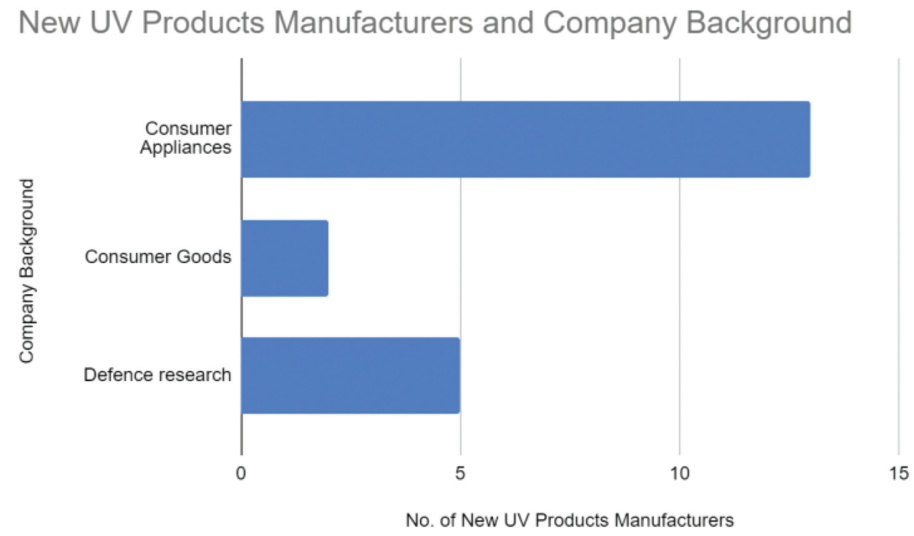

Company backgrounds of 18 new UV disinfection product manufacturers 


\section{REFERENCES}

[1] Source Google Search-COVID 19 Statistics. Retrieved July 31, 2020 from https://www.google.com/search?q=COVID+19+Statistics\& oq $=$ COVID $+19+$ Statistics.

[2] WHO. Novel Coronavirus. 2019. Retrieved July 31, 2020 from https://www.who.int/emergencies/diseases/novel-coronavirus-2019/ advice-for-public/myth-busters.

[3] Wikipedia. Travel Restrictions Related to the COVID-19 Pandemic. Retrieved July 31, 2020 from https://en.wikipedia.org/wiki/Travel_ restrictions_related_to_the_COVID-19_pandemic.

[4] Thomas Hale, Noam Angrist, Beatriz Kira, Anna Petherick, Toby Phillips, and Samuel Webster. 2020. Variation in government responses to COVID-19. BSG working paper series. BSG-WP-2020/032. Version 6.0, Blavatnik School of Government, University of Oxford.

[5] Ronald Cockx, Dieter Armbruster, and Julia C. Bendul. 2019. Resource sharing as supply chain disruption risk management measure. IFAC-PapersOnLine, 52, 13 (2019), 802-807. https://doi.org/10.1016/j.ifacol.2019.11.228

[6] C. Craighead, J. Blackhurst, M. Rungtusanatham, and R. Handfield. 2007. The severity of supply chain disruptions: Design characteristics and mitigation capabilities. F. Decis. Sci. 38, 1 (2007), 131-156. https://doi.org/10.1111/j.1540-5915.2007.00151.x

[7] Aarshi Tirkey. 2020. COVID-19: Export Bans, Trade Rules and International Cooperation. Observer Research Foundation. Retrieved from https://www.orfonline.org/expert-speak/covid19-export-bans-trade-rules-international-cooperation/.

[8] WTO Report. April 2020. Trade in Medical Goods in the Context of Tackling COVID-19. Retrieved from https://www.wto.org/english/ news_e/news20_e/rese_03apr20_e.pdf.

[9] Karl T. Ulrich and Steven D. Eppinger. 2016. Product Design and Development (5th ed.). Mc Graw-Hill Education.

[10] Wikipedia. COVID-19 Pandemic Lockdown in India. Retrieved July 31, 2020 from https://en.wikipedia.org/wiki/COVID-19_pandemic_ lockdown_in_India.

[11] PIB Delhi, Ministry of Chemicals and Fertilizers. Procuring medical equipments on priority, 01 May 2020. Retrieved July 31, 2020 from https://pib.gov.in/PressReleasePage.aspx?PRID=1620047.

[12] Ankita Sharma, Kanika Verma, and Aarushi Aggarwal. 2020. Helping India Breathe: Ventilator Manufacturing during Covid-19. Retrieved July 31, 2020 from https://www.investindia.gov.in/siru/helping-india-breathe-ventilator-manufacturing-during-covid- 19.

[13] The Economic Times. 2020. M\&M Ventilators Ready for Production and May Not Leave Factories, But Anand Mahindra Is Proud Anyway. Retrieved from https:/economictimes.indiatimes.com/magazines/panache/mm-ventilators-ready-for-production-and-may-notleave-factories-but-anand-mahindra-is-proud-anyway/articleshow/75750883.cms?.

[14] Mishika Nayyar and Remya Lakshmanan. 2020. Personal Protective Equipment in Indi. Invest India Report. Retrieved July 31, 2020 from https://static.investindia.gov.in/2020-07/PPE\%20Report_Invest\%20India.pdf.

[15] Apparel Resources News-Desk. Arvind Ltd.'s Ranchi Unit Gets Appreciation from Local Administration to NITI Aayog. May 23, 2020. Retrieved July 31, 2020 from https://in.apparelresources.com/business-news/trade/arvind-ltd-s-ranchi-unit-gets-appreciation-localadministration-niti-aayog/

[16] Kirsten Korosec and Tech Crunch. 2020. GM Delivers First Ventilators under 30,000-Unit Government Contract. Retrieved July 31, 2020 from https://techcrunch.com/2020/04/17/gm-delivers-first-ventilators-under-30000-unit-government-contract/.

[17] Ranjay Gulati. 1995. Does familiarity breed trust? The implications of repeated ties for contractual choice in alliances. Acad. Manage. 7. 38, 1 (1995) 85-112.

[18] Hiroshi Yasuda and Junichi Iijim. 2005. Linkage between strategic alliances and firm's business strategy: the case of semiconductor industry, Technovation 25, 5 (2005), 513-521. https://doi.org/10.1016/j.technovation.2003.09.005

[19] Yu Baojun, Xu Hangjun, and Dong Feng. 2019. Vertical vs. Horizontal: How strategic alliance type influence firm performance? Sustainability 11, 23 (2019), 6594. https://doi.org/10.3390/su11236594

[20] Homin Chen and Tain-Jy Chen. 2003. Governance structures in strategic alliances: transaction cost versus resource-based perspective. fournal of World Business 38, 1 (2003), 1-14. https://doi.org/10.1016/S1090-9516(02)00105-0

[21] Till Becker and Hendrik Stern. 2016. Impact of resource sharing in manufacturing on logistical key figures. Proc. CIRP 41 (2016), 579-584, ISSN 2212-8271, https://doi.org/10.1016/j.procir.2015.12.037.

[22] M. R. Chertow. 2000. Industrial symbiosis: Literature and taxonomy. Annu. Rev. Energy Environ. 25, 1 (2000) 313-337. 10.1146/annurev energy.25.1.313

[23] Luca Fraccascia, Ilaria Giannoccaro, and Vito Albin. 2017. Rethinking resilience in industrial symbiosis: Conceptualization and measurements. Ecol. Econ. 137 (2017), 148-162. https://doi.org/10.1016/j.ecolecon.2017.02.026

[24] N. B. Jacobsen. 2006. Industrial symbiosis in Kalundborg, Denmark: a quantitative assessment of economic and environmental aspects. 7. Ind. Ecol. 10, 1-2 (2006), 239-255. DOI : https://doi.org/10.1162/108819806775545411

[25] S. Evans, L. Fernando, and M. Yang. 2017. Sustainable value creation-from concept towards implementation. In Sustainable Manufacturing. Sustainable Production, Life Cycle Engineering and Management. R. Stark, G. Seliger, J. Bonvoisin (eds.). Springer, Cham. https://doi.org/10.1007/978-3-319-48514-0_13

[26] Neves Angela, Godina Radu, G. Azevedo, Susana Pimentel Carina, C. O. Matias João. 2019. The potential of industrial symbiosis: Case analysis and main drivers and barriers to its implementation. Sustainability 11, 24 (2019), 7095. https://doi.org/10.3390/su11247095

Digital Government: Research and Practice, Vol. 2, No. 2, Article 17. Publication date: December 2020. 
[27] Mônica Cavalcanti Sá de Abreu, Domenico Ceglia. 2018. On the implementation of a circular economy: The role of institutional capacity-building through industrial symbiosis. Resources, Conservation and Recycling 138 (2018), 99-109. https://doi.org/10.1016/j. resconrec.2018.07.001

[28] J. A. Mathews and H. Tan. 2011. Progress toward a circular economy in China. F. Ind. Ecol. 15, 3 (2011), 435-457. https://doi.org/10. 1111/j.1530-9290.2011.00332.x

[29] Z. Liu, M. Adams, R. P. Cote, Q. Chen, R. Wu, Z. Wen, W. Liu, and L. Dong. 2018. How does circular economy respond to greenhouse gas emissions reduction: An analysis of Chinese plastic recycling industries. Renew. Sustain.Energy Rev. 91 (2018), 1162-1169. https: //doi.org/10.1016/j.rser.2018.04.038

[30] H. Shi, M. Chertow, and Y. Song. 2010. Developing country experience with eco-industrial parks: A case study of the tianjin economictechnological development area in China. F. Clean. Prod. 18 (2010), 191-199. https://doi.org/10.1016/j.jclepro.2009.10.002

[31] R. Van Berkel, T. Fujita, S. Hashimoto, and Y. Geng. 2009. Industrial and urban symbiosis in Japan: Analysis of the eco-town program 1997-2006. 7. Environ. Manage. 90, 3 (2009), 1544-56. DOI : 10.1016/j.jenvman.2008.11.010

[32] R. Van Berkel, T. Fujita, S. Hashimoto, and M. Fujii. 2009. Quantitative assessment of urban and industrial symbiosis in Kawasaki. Jpn. Environ. Sci. Technol. 43, 5 (2009), 1271-81. DOI : 10.1021/es803319r

[33] M. Zineldin. 2004. Coopetition: the organisation of the future. Market. Intell. Plan. 22, 7 (2004), 780-790. https://doi.org/10.1108/ 02634500410568600

[34] Wikipedia. Coopetition. Retrieved July 31, 2020 from https://en.wikipedia.org/wiki/Coopetition.

[35] Erik L. Olson. 2008. The implications of platform sharing on brand value. F. Prod. Brand Manage. 17, 4 (2008), 244-53. https://doi.org/ $10.1108 / 10610420810887590$

[36] J. I. Halman, A. P. Hofer, and W. van Vuuren. 2003. Platform-driven development of product families: Linking theory with practice. 7. Prod. Innov. Manage. 20, 2 (2003), 149-162. https://doi.org/10.1111/1540-5885.2002007

[37] M. H. Meyer and J. Utterback. 1993. The product family and the dynamics of core capability. Sloan Manage. Rev. 34, 3 (1993) $29-47$.

[38] M. Uzumeri and S. Sanderson. 1995. A framework for model and product family competition. Res. Policy. 24, 4 (1995), 583-607. https: //doi.org/10.1016/S0048-7333(94)00788-8

[39] Ketan Thakkar and Ashutosh R. Shyam. 2019. Cross-badging with Toyota Hurts Maruti Baleno Sales. Times of India. October 15, 2019. Retrieved July 31, 2020 from https://timesofindia.indiatimes.com/business/india-business/cross-badging-with-toyota-hurts-marutibaleno-sales/articleshow/71598663.cms.

[40] Junming Zhu and Matthias Ruth. 2013. Exploring the resilience of industrial ecosystems. F. Environ. Manage. 122 (2013), 65-75. https: //doi.org/10.1016/j.jenvman.2013.02.052

[41] Wikipedia. Technology during World War II. Retrieved July 31, 2020 from https://en.wikipedia.org/wiki/Technology_during_World_ War_II.

Received August 2020; accepted October 2020

Digital Government: Research and Practice, Vol. 2, No. 2, Article 17. Publication date: December 2020. 